\title{
Some associative submanifolds of the squashed 7-sphere
}

\author{
Kotaro Kawai *
}

\begin{abstract}
The squashed 7-sphere $S^{7}$ is a 7 -sphere with an Einstein metric given by the canonical variation and its cone $\mathbb{R}^{8}-\{0\}$ has full holonomy $\operatorname{Spin}(7)$. There is a canonical calibrating 4 -form $\Phi$ on $\mathbb{R}^{8}-\{0\}$. A minimal 3submanifold in $S^{7}$ is called associative if its cone is calibrated by $\Phi$.

In this paper, we classify two types of fundamental associative submanifolds in the squashed $S^{7}$. One is obtained by the intersection with a 4-plane and the other is homogeneous. Then we study their infinitesimal associative deformations and explicitly show that all of them are integrable.
\end{abstract}

\section{Introduction}

A Riemannian 7-manifold $(Y, g)$ is called a nearly parallel $G_{2}$-manifold if its cone $(C(Y), \bar{g})=\left(\mathbb{R}_{>0} \times Y, d r^{2}+r^{2} g\right)$ has holonomy contained in $\operatorname{Spin}(7)$. The existence of such a structure is equivalent to that of a spin structure with a real Killing spinor ([1]), which is also used in supergravity and superstring theory in physics. There is a canonical calibrating 4-form $\Phi$ on $C(Y)$. A 3-submanifold $M$ in $Y$ is called associative if its cone $C(M)$ is Cayley, i.e. it is calibrated by $\Phi$.

By definition, Sasaki-Einstein manifolds, especially 3-Sasakian manifolds, admit nearly parallel $G_{2}$-structures. Moreover, every compact 3-Sasakian 7manifold admits a second nearly parallel $G_{2}$-structure whose cone metric has full holonomy $\operatorname{Spin}(7)(4)$. The 7 -sphere $S^{7}$ with this second nearly parallel $G_{2}$-structure is called the squashed $S^{7}$.

Associative submanifolds in the standard $S^{7}$ were studied by Lotay [7. In this paper, we study some fundamental associative submanifolds in the squashed $S^{7}$ and compare the properties.

First, we find some fundamental examples of associative submanifolds in the squashed $S^{7}$. Fibers of the Hopf fibration $\pi: S^{7} \rightarrow S^{4}$ are associative. More generally, the Hopf lifts of $I_{1}^{\prime}$-holomorphic curves in $\mathbb{C} P^{3}$ are also associative in the squashed $S^{7}$ (Proposition 4.9), where $I_{1}^{\prime}$ is an almost complex structure on $\mathbb{C} P^{3}$ given by (4.2).

Next, we classify associative submanifolds obtained by the intersection with a 4-plane. Note that the automorphism group of the squashed $S^{7}$ is $\operatorname{Sp}(1) \operatorname{Sp}(2)=$ $\mathrm{Sp}(1) \times \mathrm{Sp}(2) /\{ \pm(1,1)\}($ Lemma 4.5) .

\footnotetext{
${ }^{*}$ The author is supported by Grant-in-Aid for JSPS fellows (26-7067).
} 
Theorem 1.1. Let $V \subset \mathbb{R}^{8}=\mathbb{C}^{4}$ be a 4-plane. Suppose that $V \cap S^{7}$ is associative in the squashed $S^{7}$. Then up to the $\operatorname{Sp}(1) \operatorname{Sp}(2)$-action, $V$ is either

$V_{1}=\left\{\left(z_{1}, z_{2}, 0,0\right) \in \mathbb{C}^{4} ; z_{1}, z_{2} \in \mathbb{C}\right\} \quad$ or $\quad V_{2}=\left\{\left(z_{1}, 0, z_{3}, 0\right) \in \mathbb{C}^{4} ; z_{1}, z_{3} \in \mathbb{C}\right\}$

In other words, the space $\mathcal{M}$ of 4-planes whose intersections with $S^{7}$ are associative is described as

$$
\mathcal{M}=\operatorname{Sp}(1) \operatorname{Sp}(2) / K_{1} \sqcup \operatorname{Sp}(1) \operatorname{Sp}(2) / K_{2},
$$

where $K_{1}=\operatorname{Sp}(1)(\operatorname{Sp}(1) \times \operatorname{Sp}(1))$, and $K_{2}=\mathrm{U}(1) \mathrm{U}(2)$.

Remark 1.2. We see that $\mathcal{M}$ consists of two connected components, while the corresponding space in the standard $S^{7}$ is a homogeneous space $\operatorname{Spin}(7) / K$, where $K=\mathrm{SU}(2)^{3} / \mathbb{Z}_{2}([5])$.

Note that $V_{1}$ is a quaternionic plane in $\mathbb{C}^{4}=\mathbb{H}^{2}$ and $V_{2}$ arises from a horizontal $I_{1}$-curve of $\mathbb{C} P^{3}$ in the sense of Remark4.10, Moreover, both $V_{j} \cap S^{7}$, where $j=1,2$, are totally geodesic submanifolds in the squashed $S^{7}$. Actually, we should classify totally geodesic associative submanifolds, but it would be difficult because the squashed $S^{7}$ is neither a space of the constant curvature nor a symmetric space. It is just a homogeneous space $\operatorname{Sp}(1) \operatorname{Sp}(2) / \operatorname{Sp}(1) \operatorname{Sp}(1)$.

Next, we classify homogeneous associative submanifolds.

Theorem 1.3. Let $A$ be a connected associative 3-fold in the squashed $S^{7} \subset \mathbb{C}^{4}$ which is the orbit of a closed Lie subgroup of $\operatorname{Sp}(1) \operatorname{Sp}(2)$. Then, up to the $\operatorname{Sp}(1) \operatorname{Sp}(2)-$ action, $A$ is one of the following.

1. $L_{1}=V_{1} \cap S^{7}$, where $V_{1}$ is given in Theorem 1.1,

2. $L_{2}=V_{2} \cap S^{7}$, where $V_{2}$ is given in Theorem 1.1,

3. $A_{1}=T^{3} \cdot \frac{1}{2}^{t}(1,1,1, i) \cong T^{3}$, where the $T^{3}$-action is given by (6.1),

4. $A_{2}=\mathrm{SU}(2) \cdot{ }^{t}(1,0,0,0) \cong \mathrm{SU}(2) / \mathbb{Z}_{3}$, where the $\mathrm{SU}(2)$-action is given by (6.9),

5. $A_{3}=\mathrm{SU}(2) \cdot{ }^{t}(0,0,1,0) \cong \mathrm{SU}(2)$, where the $\mathrm{SU}(2)$-action is given by (6.9).

Remark 1.4. Since $T^{3}$ in (6.1) and $\mathrm{SU}(2)$ in (6.9) are contained in $\mathrm{SU}(4) \subset$ $\operatorname{Spin}(7)$ by an appropriate change of coordinates, we obtain the similar orbits $A_{1}, A_{2}$, and $A_{3}$ as in the standard $S^{7}$ case ([7]). However, since $G_{2}$ is not contained in $\operatorname{Sp}(1) \operatorname{Sp}(2)$, there are no corresponding associative orbits in the squashed $S^{7}$ to Lagrangian (totally real) submanifolds in $S^{6}$ classified by $[9]$.

Remark 1.5. The examples $A_{1}, A_{2}$, and $A_{3}$ are Hopf lifts of $I_{1}^{\prime}$-holomorphic curves in $\mathbb{C} P^{3}$, where $I_{1}^{\prime}$ is an almost complex structure on $\mathbb{C} P^{3}$ given by (4.2). In particular, $A_{2}$ (resp. $A_{3}$ ) is a Hopf lift of a horizontal holomorphic curve (resp. a null-torsion $I_{1}^{\prime}$-holomorphic curve defined in Definition 7.15 ) in $\mathbb{C} P^{3}$. Thus, unfortunately, we cannot find homogeneous examples which do not arise from other geometries as in the standard $S^{7}$ case ([7]). It is a further problem to find an associative submanifold which is not congruent to the fiber of $S^{7} \rightarrow S^{4}$ or the Hopf lift of an $I_{1}^{\prime}$-holomorphic curve in $\mathbb{C} P^{3}$ by the $\operatorname{Sp}(1) \operatorname{Sp}(2)$-action. As far as the author is aware, such examples are not known so far. 
However, by virtue of this property, we can explain their associative deformations.

Theorem 1.6. The associative deformations of $L_{1}, L_{2}$, and $A_{1}$ are trivial, i.e. all the associative deformations come from the $\operatorname{Sp}(1) \operatorname{Sp}(2)$-action, while $A_{2}$ and $A_{3}$ have nontrivial associative deformations.

All the associative deformations of $A_{2}$ consist of deformations of $p_{1}\left(A_{2}\right)$ as a horizontal holomorphic curve, i.e. those from the $\mathrm{PGL}(4, \mathbb{C})$-action on $\mathbb{C} P^{3}$ via the Hopf lift, and those from actions of $j, k \in \operatorname{Sp}(1)$, where $p_{1}: S^{7} \rightarrow \mathbb{C} P^{3}$ is a projection.

All the associative deformations of $A_{3}$ consist of deformations of $p_{1}\left(A_{3}\right)$ as a null-torsion holomorphic curve, and those from actions of $j, k \in \operatorname{Sp}(1)$.

Remark 1.7. The deformations of the associative submanifolds in the standard $S^{7}$ are studied by the author ([6]). We could not explain the deformation space of the associative submanifold corresponding to $A_{3}$, which did not arise from other known geometries. However, in the squashed $S^{7}$ case, the associative deformations of $A_{3}$ are explained by the property in Remark 1.5. We use the one-to-one correspondence between null-torsion $I_{1}^{\prime}$-holomorphic curves and horizontal holomorphic curves in $\mathbb{C} P^{3}([12])$.

This paper is organized as follows. In Section 2, we review the fundamental facts of $G_{2}$ and $\operatorname{Spin}(7)$ geometry. In Section 3, we review the canonical variation and summarize some useful equations. In Section 4, we apply it to the 7 -sphere $S^{7}$ and describe the nearly parallel $G_{2}$-structure on the squashed $S^{7}$ explicitly. Then we give basic examples of associative submanifolds in the squashed $S^{7}$. In Section 5, we prove Theorem 1.1 by choosing a "good" frame by $\operatorname{Sp}(1) \operatorname{Sp}(2)$ action. In Section 6, we prove Theorem 1.3 as an analogue of [7, [9]. In Section 7, we prove Theorem 1.6 by using the representation theory as [6], 10].

Acknowledgements: The author would like to thank Professor Katsuya Mashimo for his valuable advice about the representation theory.

\section{Preliminaries}

\section{$2.1 \quad G_{2}$ and $\operatorname{Spin}(7)$ geometry}

Definition 2.1. Define a 3 -form $\varphi_{0}$ on $\mathbb{R}^{7}$ by

$$
\varphi_{0}=d x_{123}+d x_{1}\left(d x_{45}+d x_{67}\right)+d x_{2}\left(d x_{46}-d x_{57}\right)-d x_{3}\left(d x_{47}+d x_{56}\right),
$$

where $\left(x_{1}, \cdots, x_{7}\right)$ is the standard coordinate on $\mathbb{R}^{7}$ and wedge signs are omitted. The Hodge dual of $\varphi_{0}$ is given by

$* \varphi_{0}=d x_{4567}+d x_{23}\left(d x_{67}+d x_{45}\right)+d x_{13}\left(d x_{57}-d x_{46}\right)-d x_{12}\left(d x_{56}+d x_{47}\right)$.

Decompose $\mathbb{R}^{8}=\mathbb{R} \oplus \mathbb{R}^{7}$ and denote by $x_{0}$ the coordinate on $\mathbb{R}$. Define a self-dual 4 -form $\Phi_{0}$ on $\mathbb{R}^{8}$ by

$$
\Phi_{0}=d x_{0} \wedge \varphi_{0}+* \varphi_{0} .
$$

If we identify $\mathbb{R}^{8} \cong \mathbb{C}^{4}$ via $\mathbb{R}^{8} \ni\left(x_{0}, \cdots, x_{7}\right) \mapsto\left(x_{0}+i x_{1}, x_{2}+i x_{3}, x_{4}+i x_{5}, x_{6}+\right.$ $\left.i x_{7}\right)=:\left(z_{1}, z_{2}, z_{3}, z_{4}\right) \in \mathbb{C}^{4}$, then $\Phi_{0}$ is described as

$$
\Phi_{0}=\frac{1}{2} \omega_{0} \wedge \omega_{0}+\operatorname{Re} \Omega_{0}
$$


where $\omega_{0}=\frac{i}{2} \sum_{j=1}^{4} d z_{j \bar{j}}$ and $\Omega_{0}=d z_{1234}$ are the standard Kähler form and the holomorphic volume form on $\mathbb{C}^{4}$, respectively.

The stabilizers of $\varphi_{0}$ and $\Phi_{0}$ are the exceptional Lie group $G_{2}$ and $\operatorname{Spin}(7)$, respectively:

$G_{2}=\left\{g \in G L(7, \mathbb{R}) ; g^{*} \varphi_{0}=\varphi_{0}\right\}, \quad \operatorname{Spin}(7)=\left\{g \in G L(8, \mathbb{R}) ; g^{*} \Phi_{0}=\Phi_{0}\right\}$.

The Lie group $G_{2}$ fixes the standard metric $g_{0}=\sum_{i=1}^{7}\left(d x_{i}\right)^{2}$ and the orientation on $\mathbb{R}^{7}$. They are uniquely determined by $\varphi_{0}$ via

$$
6 g_{0}\left(v_{1}, v_{2}\right) \operatorname{vol}_{g_{0}}=i\left(v_{1}\right) \varphi_{0} \wedge i\left(v_{2}\right) \varphi_{0} \wedge \varphi_{0},
$$

where $\operatorname{vol}_{g_{0}}$ is a volume form of $g_{0}, i(\cdot)$ is the interior product, and $v_{i} \in T\left(\mathbb{R}^{7}\right)$.

Similarly, $\operatorname{Spin}(7)$ fixes the standard metric $h_{0}=\sum_{i=0}^{7}\left(d x_{i}\right)^{2}$ and the orientation on $\mathbb{R}^{8}$. They are uniquely determined by $\Phi_{0}$ via

$$
\Phi_{0}^{2}=14 \operatorname{vol}_{h_{0}}, \quad\left(i\left(w_{2}\right) i\left(w_{1}\right) \Phi_{0}\right)^{2} \wedge \Phi_{0}=6\left\|w_{1} \wedge w_{2}\right\|_{h_{0}}^{2} \operatorname{vol}_{h_{0}},
$$

where $\operatorname{vol}_{h_{0}}$ is a volume form of $h_{0}$, and $w_{i} \in T\left(\mathbb{R}^{8}\right)$.

Definition 2.2. Let $Y$ be an oriented 7-manifold and $\varphi$ a 3-form on $Y$. A 3 -form $\varphi$ is called a $G_{2}$-structure on $Y$ if for each $y \in Y$, there exists an oriented isomorphism between $T_{y} Y$ and $\mathbb{R}^{7}$ identifying $\varphi_{y}$ with $\varphi_{0}$. From (2.1), $\varphi$ induces the metric $g$ and the volume form on $Y$. A $G_{2}$-structure $\varphi$ is said to be nearly parallel if $d \varphi=4 * \varphi$. We call a manifold with a nearly parallel $G_{2}$-structure a nearly parallel $G_{2}$-manifold for short. A $G_{2}$-structure $\varphi$ is called torsion-free if $d \varphi=0, d * \varphi=0$.

Let $X$ be an oriented 8-manifold and $\Phi$ a 4-form on $X$. A 4-form $\Phi$ is called a $\operatorname{Spin}(7)$-structure on $X$ if for each $x \in X$, there exists an oriented isomorphism between $T_{x} X$ and $\mathbb{R}^{8}$ identifying $\Phi_{x}$ with $\Phi_{0}$. From (2.2), $\Phi$ induces the metric $h$ and the volume form on $X$. A $\operatorname{Spin}(7)$-structure $\Phi$ is called torsion-free if $d \Phi=0$.

Lemma 2.3. 111] $A G_{2}$-structure $\varphi$ is torsion-free if and only if $\operatorname{Hol}(g) \subset G_{2}$. $A$ Spin(7)-structure $\Phi$ is torsion-free if and only if $\operatorname{Hol}(h) \subset \operatorname{Spin}(7)$.

Lemma 2.4. The 3-form $\varphi$ is a nearly parallel $G_{2}$-structure if and only if its Riemannian cone $C(Y)=\mathbb{R}_{>0} \times Y$ admits a torsion-free $\operatorname{Spin}(7)$-structure $\Phi=r^{3} d r \wedge \varphi+r^{4} * \varphi$ with the induced cone metric $\bar{g}=d r^{2}+r^{2} g$.

Next, we give a summary of the facts about the submanifolds. Let $Y$ be a manifold with a $G_{2}$-structure $\varphi$ and the induced metric $g$.

Lemma 2.5. [5] For every oriented $k$-dimensional subspace $V^{k} \subset T_{p} Y(\forall p \in$ $Y, k=3,4)$, we have $\left.\varphi\right|_{V^{3}} \leq \operatorname{vol}_{V^{3}},\left.* \varphi\right|_{V^{4}} \leq \operatorname{vol}_{V^{4}}$. An oriented 3-submanifold $L^{3} \subset Y$ is called associative if $\left.\varphi\right|_{T L^{3}}=\operatorname{vol}_{L^{3}}$. An oriented 4-submanifold $L^{4}$ is called coassociative if $\left.* \varphi\right|_{T L^{4}}=\operatorname{vol}_{L^{4}}$.

Lemma 2.6. [5] An oriented 3-submanifold $L^{3}$ is associative if and only if $* \varphi\left(v_{1}, v_{2}, v_{3}, \cdot\right)=0$ for any $v_{j} \in T L^{3}$. An oriented 4 -submanifold $L^{4}$ is coassociative if and only if $\left.\varphi\right|_{T L^{4}}=0$. 
Remark 2.7. Define the cross product $\times: T Y \times T Y \rightarrow T Y$ by

$$
g(u \times v, w)=\varphi(u, v, w)
$$

for $u, v, w \in T Y$. When $L^{3}$ is associative, there exists an orthonormal basis $\left\{e_{1}, e_{2}, e_{3}\right\}$ satisfying $e_{3}=e_{1} \times e_{2}$ at any point in $L^{3}$.

Definition 2.8. Let $X$ be a manifold with a $\operatorname{Spin}(7)$-structure $\Phi$. Then for every oriented 4-dimensional subspace $W \subset T_{x} X(\forall x \in X)$, we have $\left.\Phi\right|_{W} \leq \operatorname{vol}_{W}$. An oriented 4-submanifold $N \subset X$ is called Cayley if $\left.\Phi\right|_{T N}=\operatorname{vol}_{N}$.

Lemma 2.9. Let $(Y, \varphi, g)$ be a nearly parallel $G_{2}$-manifold and $L \subset Y$ be an oriented 3-submanifold. By Lemma 2.4. $C(Y)$ is a manifold with a torsion-free $\operatorname{Spin}(7)$-structure $\Phi$. Then $L \subset Y$ is associative if and only if $C(L) \subset C(Y)$ is Cayley.

Lemma 2.10. [7] There are no coassociative submanifolds of a nearly parallel $G_{2}$-manifold $(Y, \varphi, g)$.

Proof. If $L$ is a coassociative submanifold, we have $\left.\varphi\right|_{T L}=0$, which implies that $4 \mathrm{vol}_{L}=\left.4 * \varphi\right|_{T L}=\left.d \varphi\right|_{T L}=0$. This is a contradiction.

\section{Canonical variation}

\subsection{Riemannian submersion}

We give a summary of Chapter 9 of [2]. Let $(M, g)$ and $(B, h)$ be Riemannian manifolds and suppose that there exists a Riemannian submersion $\pi:(M, g) \rightarrow$ $(B, h)$. Decompose the tangent bundle $T M=\mathcal{V} \oplus \mathcal{H}$, where a vertical distribution $\mathcal{V}$ is a vector subbundle tangent to the fibers $\pi: M \rightarrow B$, and a horizontal distribution $\mathcal{H}$ is the orthogonal complement bundle of $\mathcal{V}$. Denote by $\nabla$ the Levi-Civita connection of $g$.

Definition 3.1. Define $(1,2)$-tensors $A, T \in C^{\infty}\left(M, \otimes^{2} T^{*} M \otimes T M\right)$ by

$$
A_{E} F=\left(\nabla_{E^{\top}} F^{\perp}\right)^{\top}+\left(\nabla_{E^{\top}} F^{\top}\right)^{\perp}, \quad T_{E} F=\left(\nabla_{E^{\perp}} F^{\perp}\right)^{\top}+\left(\nabla_{E^{\perp}} F^{\top}\right)^{\perp},
$$

for $E, F \in \mathfrak{X}(M)$, where $\top: T M \rightarrow \mathcal{H}$ and $\perp: T M \rightarrow \mathcal{V}$ are projections.

Remark 3.2. The distribution $\mathcal{H}$ is involutive if and only if $A \equiv 0$. The fibers of $\pi: M \rightarrow B$ are totally geodesic if and only if $T \equiv 0$.

In the following, we suppose that $\underline{T \equiv 0}$.

Lemma 3.3. Let $X, Y$ be the horizontal vector fields, $U, V$ be the vertical vector fields, and $E, F$ be any vector fields on $M$. We have

$$
\begin{array}{lll}
A_{U} X=0, & A_{U} V=0, & A_{X} U=\left(\nabla_{X} U\right)^{\top}, \quad A_{X} Y=\left(\nabla_{X} Y\right)^{\perp}, \\
A_{X} Y=-A_{Y} X, & A_{X} Y=\frac{1}{2}[X, Y]^{\perp}, & g\left(A_{X} E, F\right)=-g\left(E, A_{X} F\right) .
\end{array}
$$

which implies that

$$
\begin{array}{ll}
\nabla_{U} V=\left(\nabla_{U} V\right)^{\perp}, & \nabla_{U} X=\left(\nabla_{U} X\right)^{\top}, \\
\nabla_{X} U=\left(\nabla_{X} U\right)^{\perp}+A_{X} U, & \nabla_{X} Y=A_{X} Y+\left(\nabla_{X} Y\right)^{\top} .
\end{array}
$$




\subsection{Canonical Variation}

For $s, t>0$, define the canonical variation $\tilde{g}$ of the Riemannian metric $g$ on $M$ by

$$
\left.\tilde{g}\right|_{\mathcal{V} \times \mathcal{V}}=\left.s^{2} g\right|_{\mathcal{V} \times \mathcal{V}},\left.\quad \tilde{g}\right|_{\mathcal{H} \times \mathcal{H}}=\left.t^{2} g\right|_{\mathcal{H} \times \mathcal{H}},\left.\quad \tilde{g}\right|_{\mathcal{H} \times \mathcal{V}}=0 .
$$

Remark 3.4. Usually, we set $t=1$ for simplicity. However, we introduce a parameter $t$ to define the nearly parallel $G_{2}$-structure. See Proposition 4.3 .

Denote by $\tilde{\nabla}$ the Levi-Civita connection of $\tilde{g}$. Set $(1,2)$-tensors $\tilde{A}$ and $\tilde{T}$ as in Definition 3.1 .

Remark 3.5. The assumption $T \equiv 0$ implies that $\tilde{T} \equiv 0$ for all $s, t>0$.

Under the canonical variation, the tensor $A$ in Definition 3.1 and the LeviCivita connection are changed as follows.

Lemma 3.6. Let $X, Y$ be the horizontal vector fields, and $U, V$ be the vertical vector fields on $M$. We have

$$
\begin{array}{cc}
\tilde{A}_{X} Y=A_{X} Y, & \tilde{A}_{X} U=\frac{s^{2}}{t^{2}} A_{X} U, \\
\tilde{\nabla}_{X} Y=\nabla_{X} Y, & \tilde{\nabla}_{U} V=\nabla_{U} V, \\
\tilde{\nabla}_{X} U=\frac{s^{2}}{t^{2}}\left(\nabla_{X} U\right)^{\top}+\left(\nabla_{X} U\right)^{\perp}, \\
\tilde{\nabla}_{U} X=\frac{s^{2}}{t^{2}}\left(\nabla_{U} X\right)^{\top}+\left(1-\frac{s^{2}}{t^{2}}\right)[U, X]^{\top} .
\end{array}
$$

This lemma implies the following useful equation.

Lemma 3.7. For $E_{1}, E_{2} \in \mathfrak{X}(M)$, we have

$$
\tilde{\nabla}_{E_{1}} E_{2}-\nabla_{E_{1}} E_{2}=\left(-1+\frac{s^{2}}{t^{2}}\right)\left(A_{E_{1}} E_{2}^{\perp}+A_{E_{2}} E_{1}^{\perp}\right) \text {. }
$$

\section{Nearly parallel $G_{2}$-structure on the squashed $S^{7}$}

The standard $S^{7}$ admits a canonical nearly parallel $G_{2}$-structure. By the canonical variation, we obtain the second nearly parallel $G_{2}$-structure on $S^{7}$ (Proposition 4.3). First, we review a 3-Sasakian structure on $S^{7}$.

\section{$4.1 \quad 3$-Sasakian structure on $S^{7}$}

Consider the following Lie groups:

$$
\begin{aligned}
\operatorname{Sp}(1) & =\left\{a_{1}+a_{2} j \in \mathbb{H} ; a_{i} \in \mathbb{C},\left|a_{1}\right|^{2}+\left|a_{2}\right|^{2}=1\right\}, \\
\operatorname{Sp}(2) & =\left\{g \in \mathrm{GL}(2, \mathbb{H}) ; g \text { preserves the metric on } \mathbb{H}^{2}\right\} \\
& =\left\{g \in \mathrm{U}(4) ;{ }^{t} g J g=J\right\} \\
& =\left\{(u, J \bar{u}, v, J \bar{v}) ; u, v \in \mathbb{C}^{4},|u|=|v|=1,\langle v, u\rangle_{\mathbb{C}}=\langle v, J \bar{u}\rangle_{\mathbb{C}}=0\right\},
\end{aligned}
$$


where $J=\left(\begin{array}{cc}J^{\prime} & 0 \\ 0 & J^{\prime}\end{array}\right), J^{\prime}=\left(\begin{array}{cc}0 & -1 \\ 1 & 0\end{array}\right)$, and $\langle\cdot, \cdot\rangle_{\mathbb{C}}: \mathbb{C}^{4} \times \mathbb{C}^{4} \rightarrow \mathbb{C}$ is the standard Hermitian metric on $\mathbb{C}^{4}$.

Let $\operatorname{Sp}(1) \times \operatorname{Sp}(2)$ act on $\mathbb{H}^{2}$ by

$$
(q, A) \cdot\left(q_{1}, q_{2}\right)=q\left(q_{1}, q_{2}\right)^{\bar{A}},
$$

where $(q, A) \in \mathrm{Sp}(1) \times \mathrm{Sp}(2),\left(q_{1}, q_{2}\right) \in \mathbb{H}^{2}$. Via the identification $\mathbb{C}^{4} \ni\left(z_{1}, \cdots, z_{4}\right) \mapsto$ $\left(z_{1}+z_{2} j, z_{3}+z_{4} j\right) \in \mathbb{H}^{2}$, the $\operatorname{Sp}(1)$-action on $\mathbb{C}^{4}$ is described as

$$
\left(a_{1}+a_{2} j\right) \cdot u=a_{1} u+a_{2} J \bar{u},
$$

where $u \in \mathbb{C}^{4}$, and $\operatorname{Sp}(2) \subset \mathrm{U}(4)$ acts on $\mathbb{C}^{4}$ canonically. By definition, the $\operatorname{Sp}(1)$-action commutes with the $\operatorname{Sp}(2)$-action.

The actions of $i, j, k \in \mathrm{Sp}(1)$ induce complex structures $I_{1}, I_{2}, I_{3}$ on $\mathbb{C}^{4}$, respectively, and hence induce the 3-Sasakian structure $\left\{\left(\Phi_{i}, \xi_{i}, \eta_{i}, g\right)\right\}_{i=1,2,3}$ on $S^{7}$, where $g$ is the standard metric on $S^{7}$, and a vector field $\xi_{i} \in \mathfrak{X}\left(S^{7}\right)$, a 1-form $\eta_{i} \in \Omega^{1}\left(S^{7}\right)$, and a $(1,1)$-tensor $\Phi_{i} \in C^{\infty}\left(S^{7}, \operatorname{End}\left(T S^{7}\right)\right)$ are defined by

$$
\begin{aligned}
\left(\xi_{i}\right)_{z} & =-I_{i}(z), \text { where } z \in \mathbb{C}^{4}, \\
\eta_{i} & =g\left(\xi_{i}, \cdot\right), \\
\Phi_{i} & = \begin{cases}I_{i} & \left(\text { on Ker } \eta_{i}\right) \\
0 & \left(\text { on } \mathbb{R} \xi_{i}\right) .\end{cases}
\end{aligned}
$$

Note that the following conditions are satisfied:

$$
\begin{aligned}
\Phi_{i+2} & =\Phi_{i} \circ \Phi_{i+1}-\eta_{i+1} \otimes \xi_{i}=-\Phi_{i+1} \circ \Phi_{i}+\eta_{i} \otimes \xi_{i+1}, \\
\xi_{i+2} & =\Phi_{i}\left(\xi_{i+1}\right)=-\Phi_{i+1}\left(\xi_{i}\right), \\
\eta_{i+2} & =\eta_{i} \circ \Phi_{i+1}=-\eta_{i+1} \circ \Phi_{i},
\end{aligned}
$$

where $i \in \mathbb{Z} / 3$. These tensors are described explicitly as follows.

\section{Lemma 4.1 .}

$$
\begin{gathered}
\xi_{1}=-i^{t}\left(z_{1}, z_{2}, z_{3}, z_{4}\right), \\
\xi_{2}={ }^{t}\left(\bar{z}_{2},-\bar{z}_{1}, \bar{z}_{4},-\bar{z}_{3}\right), \\
\xi_{3}=i^{t}\left(\bar{z}_{2},-\bar{z}_{1}, \bar{z}_{4},-\bar{z}_{3}\right), \\
\eta_{1}=\operatorname{Im}\left(\sum_{j=1}^{4} z_{j} d \bar{z}_{j}\right), \quad \eta_{2}+i \eta_{3}=-z_{1} d z_{2}+z_{2} d z_{1}-z_{3} d z_{4}+z_{4} d z_{3}, \\
d \eta_{1}=-i \sum_{j=1}^{4} d z_{j \bar{j}}=-2 g\left(\Phi_{1}(\cdot), \cdot\right), \quad d\left(\eta_{2}+i \eta_{3}\right)=-2\left(d z_{12}+d z_{34}\right) .
\end{gathered}
$$

\subsection{Second nearly parallel $G_{2}$-structure on $S^{7}$}

Applying the canonical variation to a Riemannian submersion $\pi: S^{7} \rightarrow S^{4}=$ $\mathbb{H} P^{1}$, we obtain the second nearly parallel $G_{2}$-structure $(\tilde{\varphi}, \tilde{g})$ on $S^{7}$. Denote 
by $\omega_{i}=\frac{1}{2} D \eta_{i}=\frac{1}{2} d \eta_{i}\left((\cdot)^{\top},(\cdot)^{\top}\right) \in \Omega^{2}\left(S^{7}\right)$ the covariant differentiation of $\frac{1}{2} \eta_{i}$, where $T: T S^{7} \rightarrow \mathcal{H}$ is a canonical projection. In other words, we have

$$
\omega_{1}=\frac{1}{2} d \eta_{1}+\eta_{23}, \quad \omega_{2}=\frac{1}{2} d \eta_{2}+\eta_{31}, \quad \omega_{3}=\frac{1}{2} d \eta_{3}+\eta_{12} .
$$

since $\left[\xi_{i}, \xi_{i+1}\right]=2 \xi_{i+2}$ for $i \in \mathbb{Z} / 3$. On the other hand, it is well-known that $\frac{1}{2} d \eta_{i}=-g\left(\Phi_{i}(\cdot), \cdot\right)$. For example, see Section 2 of [10]. Then we deduce that

$$
\omega_{i}=-g\left(\Phi_{i}(\cdot)^{\top},(\cdot)^{\top}\right) \quad \text { for } i=1,2,3 .
$$

Remark 4.2. Take any unit vector $X_{0} \in \mathcal{H}$ and set $X_{i}=\Phi_{i}\left(X_{0}\right)$ for $i=1,2,3$. Denote by $\left\{X^{i}\right\}$ the dual of $\left\{X_{i}\right\}$. Then we have

$$
\omega_{1}=-\left(X^{01}+X^{23}\right), \quad \omega_{2}=-\left(X^{02}+X^{31}\right), \quad \omega_{3}=-\left(X^{03}+X^{12}\right) .
$$

Proposition 4.3. 4] Define the Riemannian metric $\tilde{g}$, a 3-form $\tilde{\varphi} \in \Omega^{3}\left(S^{7}\right)$, and the 4 -form $* \tilde{\varphi} \in \Omega^{4}\left(S^{7}\right)$ on $S^{7}$ by

$$
\begin{aligned}
\left.\tilde{g}\right|_{\mathcal{V} \times \mathcal{V}} & =\left.\left(\frac{3}{5}\right)^{2} g\right|_{\mathcal{V} \times \mathcal{V}},\left.\quad \tilde{g}\right|_{\mathcal{H} \times \mathcal{H}}=\left.\left(\frac{3}{\sqrt{5}}\right)^{2} g\right|_{\mathcal{H} \times \mathcal{H}},\left.\quad \tilde{g}\right|_{\mathcal{H} \times \mathcal{V}}=0, \\
\tilde{\varphi} & =\frac{27}{25}\left(\frac{1}{5} \eta_{123}+\sum_{i=1}^{3} \eta_{i} \wedge \omega_{i}\right), \\
* \tilde{\varphi} & =\frac{27}{25}\left(\frac{1}{2} \sum_{i=1}^{3} \omega_{i}^{2}+\frac{3}{5}\left(\eta_{23} \wedge \omega_{1}+\eta_{31} \wedge \omega_{2}+\eta_{12} \wedge \omega_{3}\right)\right) .
\end{aligned}
$$

Then $(\tilde{\varphi}, \tilde{g})$ is a nearly parallel $G_{2}$-structure with $\operatorname{Hol}(\overline{\tilde{g}})=\operatorname{Spin}(7)$ and $* \tilde{\varphi}$ is a Hodge dual of $\tilde{\varphi}$ with respect to $\tilde{g}$. We call $\left(S^{7}, \tilde{\varphi}, \tilde{g}\right)$ the squashed $S^{7}$.

Outline of the proof. Set

$$
\begin{gathered}
\left.\tilde{g}\right|_{\mathcal{V} \times \mathcal{V}}=\left.s^{2} g\right|_{\mathcal{V} \times \mathcal{V}},\left.\quad \tilde{g}\right|_{\mathcal{H} \times \mathcal{H}}=\left.t^{2} g\right|_{\mathcal{H} \times \mathcal{H}},\left.\quad \tilde{g}\right|_{\mathcal{H} \times \mathcal{V}}=0, \\
\tilde{\varphi}=s^{3} \eta_{123}+s t^{2} \sum_{i=1}^{3} \eta_{i} \wedge \omega_{i},
\end{gathered}
$$

for $s, t>0$. We find $s, t>0$ satisfying $d \tilde{\varphi}=4 * \tilde{\varphi}$. Setting $G_{1}=s^{3} \eta_{123}, G_{2}=$ $s t^{2} \sum_{i=1}^{3} \eta_{i} \wedge \omega_{i}$, we have

$$
\begin{aligned}
& * G_{1}=\frac{t^{4}}{6} \sum_{i=1}^{3} \omega_{i}^{2}, \quad * G_{2}=s^{2} t^{2}\left(\eta_{23} \wedge \omega_{1}+\eta_{31} \wedge \omega_{2}+\eta_{12} \wedge \omega_{3}\right), \\
& d\left(\eta_{123}\right)=\frac{2}{s^{2} t^{2}} * G_{2}, \quad d\left(\sum_{i=1}^{3} \eta_{i} \wedge \omega_{i}\right)=\frac{12}{t^{4}} * G_{1}+\frac{2}{s^{2} t^{2}} * G_{2} .
\end{aligned}
$$

Then we see that $d \tilde{\varphi}=\frac{12}{s} * G_{1}+\left(\frac{2 s}{t^{2}}+\frac{2}{s}\right) * G_{2}$, and hence $d \tilde{\varphi}=4 * \tilde{\varphi}$ is equivalent to $s=3 / 5, t=3 / \sqrt{5}$. The metric $\tilde{g}$ is not Sasaki-Einstein, and hence satisfies $\operatorname{Hol}(\overline{\tilde{g}})=\operatorname{Spin}(7)$ by the classification of the dimensions of the spaces of real Killing spinors. 
Remark 4.4. 4] Proposition 4.3 is valid for any compact 3-Sasakian manifolds. The metric $\tilde{g}$ is Einstein if and only if $s=t$ or $s=t / \sqrt{5}$.

Since $\eta_{1}=\operatorname{Im}\left({ }^{t} z d \bar{z}\right), \eta_{2}+i \eta_{3}=-d^{t} z \cdot J z$, where $z={ }^{t}\left(z_{1}, z_{2}, z_{3}, z_{4}\right), \operatorname{Sp}(2)$ preserves $\eta_{j}(j=1,2,3)$. For $q=a_{1}+a_{2} j \in \operatorname{Sp}(1)$, we have $\left(q^{*} \eta_{1}, q^{*} \eta_{2}, q^{*} \eta_{3}\right)=$ $\left(\eta_{1}, \eta_{2}, \eta_{3}\right)^{t} M_{q}$, where $M_{q} \in \mathrm{SO}(3)$ is described as

$$
M_{q}=\left(\begin{array}{ccc}
\left|a_{1}\right|^{2}-\left|a_{2}\right|^{2} & 2 \operatorname{Im}\left(a_{1} \bar{a}_{2}\right) & 2 \operatorname{Re}\left(a_{1} \bar{a}_{2}\right) \\
2 \operatorname{Im}\left(a_{1} a_{2}\right) & \operatorname{Re}\left(a_{1}^{2}+a_{2}^{2}\right) & \operatorname{Im}\left(-a_{1}^{2}+a_{2}^{2}\right) \\
-2 \operatorname{Re}\left(a_{1} a_{2}\right) & \operatorname{Im}\left(a_{1}^{2}+a_{2}^{2}\right) & \operatorname{Re}\left(a_{1}^{2}-a_{2}^{2}\right)
\end{array}\right) .
$$

Hence we see that $\operatorname{Sp}(2)$ and $\operatorname{Sp}(1)$ preserve $\left.g\right|_{\mathcal{H} \times \mathcal{H}},\left.g\right|_{\mathcal{V} \times \mathcal{V}}, \tilde{g}$ and $\tilde{\varphi}$. In fact, we have the following.

Lemma 4.5. 4] The automorphism group of the squashed $\left(S^{7}, \tilde{\varphi}, \tilde{g}\right)$ is $\operatorname{Sp}(1) \operatorname{Sp}(2)=$ $\mathrm{Sp}(1) \times \mathrm{Sp}(2) /\{ \pm(1,1)\}$.

Remark 4.6. In this paper, we often consider the subgroup of $\operatorname{Sp}(1) \operatorname{Sp}(2)$. If there may be some confusion, denoting $\operatorname{Sp}(1)=\operatorname{Sp}(1)_{L}$ and $\operatorname{Sp}(2)=\operatorname{Sp}(2)_{R}$, we distinguish subgroups of $\operatorname{Sp}(1) \operatorname{Sp}(2)=\operatorname{Sp}(1)_{L} \operatorname{Sp}(2)_{R}$.

Lemma 4.7. For any $E_{1}, E_{2} \in \mathfrak{X}\left(S^{7}\right)$, we have

$$
\begin{aligned}
\tilde{g}\left(E_{1}, E_{2}\right) & =-\frac{36}{25} \sum_{j=1}^{3} \eta_{j}\left(E_{1}\right) \eta_{j}\left(E_{2}\right)+\frac{9}{5} g\left(E_{1}, E_{2}\right), \\
\tilde{\nabla}_{E_{1}} E_{2}-\nabla_{E_{1}} E_{2} & =\frac{4}{5} \Theta\left(E_{1}, E_{2}\right),
\end{aligned}
$$

where $\Theta \in C^{\infty}\left(S^{7}, \otimes^{2} T^{*} S^{7}\right)$ is defined by

$$
\Theta\left(E_{1}, E_{2}\right)=\sum_{i=1}^{3}\left(\eta_{i}\left(E_{1}\right) \Phi_{i}\left(E_{2}\right)+\eta_{i}\left(E_{2}\right) \Phi_{i}\left(E_{1}\right)\right) .
$$

Proof. The first equation is proved easily and we omit the proof. Set $(s, t)=$ $(3 / 5,3 / \sqrt{5})$ in Lemma 3.7. Since $A_{X} U=-\sum_{i=1}^{3} \eta_{i}(U) \Phi_{i}(X)$ for a horizontal vector $X$ and a vertical vector $U$, we have

$$
\tilde{\nabla}_{E_{1}} E_{2}-\nabla_{E_{1}} E_{2}=\frac{4}{5} \sum_{i=1}^{3}\left(\eta_{i}\left(E_{1}\right) \Phi_{i}\left(E_{2}^{\top}\right)+\eta_{i}\left(E_{2}\right) \Phi_{i}\left(E_{1}^{\top}\right)\right) .
$$

We easily see that the right hand side is equal to $\frac{4}{5} \Theta\left(E_{1}, E_{2}\right)$.

\subsection{Associative submanifolds of the squashed $S^{7}$}

By the definition of $\tilde{\varphi}$ in Proposition 4.3, we see the following.

Remark 4.8. There are no horizontal associative submanifolds, i.e. there are no associative submanifolds whose tangent spaces are contained in $\mathcal{H}$.

Let $\pi: S^{7} \rightarrow S^{4}$ and $p_{1}: S^{7} \rightarrow \mathbb{C} P^{3}$ be the Hopf fibrations and $p_{2}: \mathbb{C} P^{3} \rightarrow$ $S^{4}$ be the twistor fibration satisfying $\pi=p_{2} \circ p_{1}$. Denote by $\underline{\mathcal{V}}$ and $\underline{\mathcal{H}}$ the distributions of $\mathbb{C} P^{3}$ induced by $\mathcal{V}$ and $\mathcal{H}$, respectively. In other words, $\underline{\mathcal{V}}$ is 
a vector subbundle of $T \mathbb{C} P^{3}$ tangent to the fibers $p_{2}$, and $\underline{\mathcal{H}}$ is the orthogonal complement bundle of $\underline{\mathcal{V}}$. By an abuse of notation, denote by $I_{1}$ the standard complex structure on $\mathbb{C} P^{3}$ induced from the standard complex structure $I_{1}$ on $\mathbb{C}^{4}$. Define the almost complex structure $I_{1}^{\prime}$ on $\mathbb{C} P^{3}$ by

$$
\left.I_{1}^{\prime}\right|_{\underline{\mathcal{V}}}=-\left.I_{1}\right|_{\underline{\mathcal{V}}},\left.\quad I_{1}^{\prime}\right|_{\underline{\mathcal{H}}}=\left.I_{1}\right|_{\underline{\mathcal{H}}} .
$$

The almost complex structure $I_{1}^{\prime}$ is never integrable, and defines the nearly Kähler structure on $\mathbb{C} P^{3}$.

Proposition 4.9. Let $\Sigma \subset \mathbb{C} P^{3}$ be an $I_{1}^{\prime}$-holomorphic curve. Then the Hopf lift $p_{1}^{-1}(\Sigma) \subset S^{7}$ of $\Sigma$ is associative in the squashed $S^{7}$.

Proof. Use the notation of Remark4.2 and Proposition 4.3. Setting $\tilde{\eta}_{i}=(3 / 5) \eta_{i}$ and $\tilde{X}^{i}=(3 / \sqrt{5}) X^{i}$, we have

$$
\tilde{\varphi}=\tilde{\eta}_{1}\left(\tilde{\eta}_{23}-\tilde{X}^{01}-\tilde{X}^{23}\right)-\tilde{\eta}_{2}\left(\tilde{X}^{02}+\tilde{X}^{31}\right)-\tilde{\eta}_{3}\left(\tilde{X}^{03}+\tilde{X}^{12}\right) .
$$

Then we obtain $\tilde{\eta}_{23}-\tilde{X}^{01}-\tilde{X}^{23}=-\tilde{G}\left(I_{1}^{\prime}(\cdot), \cdot\right)$, where $\tilde{G}=\tilde{\eta}_{2} \otimes \tilde{\eta}_{2}+\tilde{\eta}_{3} \otimes \tilde{\eta}_{3}+$ $\sum_{j=0}^{3} \tilde{X}^{j} \otimes \tilde{X}^{j}$, which gives the proof.

Remark 4.10. Each fiber $F \cong S^{2}$ of $p_{2}$ is an obvious $I_{1}^{\prime}$-holomorphic curve. Then the Hopf lift $p_{1}^{-1}(F)=\pi^{-1}(*)$ of $F$ is associative. This is the intersection of a quaternionic plane and $S^{7}$.

If $\Sigma \subset \mathbb{C} P^{3}$ is a horizontal $I_{1}$-holomorphic curve, where we call the curve $\Sigma$ horizontal if $\left.T \Sigma \subset \underline{\mathcal{H}}\right|_{\Sigma}, \Sigma \subset \mathbb{C} P^{3}$ is also an $I_{1}^{\prime}$-holomorphic curve. Thus the Hopf lift $p_{1}^{-1}(\Sigma)$ is associative. Since $I_{1}$ is the standard complex structure, we know many examples of these curves.

\section{Classification of Cayley planes}

In this section, we prove Theorem 1.1 Let $V^{4} \subset \mathbb{R}^{8}$ be a 4 -plane. We classify the associative submanifolds of the form $V \cap S^{7}$ by choosing a "good" frame of $V$ by the $\operatorname{Sp}(1) \operatorname{Sp}(2)$-action to consider the associative condition.

Suppose that $V$ is spanned by $e_{0}, \cdots, e_{3}\left(e_{i} \in \mathbb{C}^{4}=\mathbb{R}^{8}\right)$. Since $\operatorname{Sp}(1) \operatorname{Sp}(2)$ acts transitively on $S^{7}$, we may assume that

$$
e_{0}={ }^{t}(1,0,0,0) \text {. }
$$

The stabilizer of $\operatorname{Sp}(1) \operatorname{Sp}(2)$ at $e_{0}$ is diffeomorphic to $\operatorname{Sp}(1) \operatorname{Sp}(1)$, which acts on $S^{7}$ as $[(p, q)] \cdot\left(q_{1}, q_{2}\right)=\left(p q_{1} \bar{p}, p q_{2} \bar{q}\right)$, where $[(p, q)] \in \operatorname{Sp}(1) \operatorname{Sp}(1)$ and $\left(q_{1}, q_{2}\right) \in$ $S^{7} \subset \mathbb{H}^{2}=\mathbb{R}^{8}$. Thus we may assume that

$$
e_{1}={ }^{t}(c i, 0, s, 0)
$$

for $c, s \geq 0, c^{2}+s^{2}=1$. Since $\{[(z, z)] ; z \in \mathrm{U}(1)\} \subset \operatorname{Sp}(1) \operatorname{Sp}(1)$ fixes $e_{1}$, by sweeping out the first entry, we may assume that

$$
e_{2}={ }^{t}\left(0, A_{2}, A_{3}+i B_{3}, A_{4}+i B_{4}\right),
$$

for $A_{j}, B_{k} \in \mathbb{R}, A_{2} \geq 0$. 
Lemma 5.1. We have

$\frac{5}{3}\left(e_{1} \times e_{2}\right)_{e_{0}}={ }^{t}\left(5 B_{3} s i, 5 A_{4} s+\left(-A_{2} c+5 B_{4} s\right) i,-B_{3} c+A_{3} c i,\left(-B_{4} c-A_{2} s\right)+A_{4} c i\right)$.

Thus denoting by $e_{3}$ the left-hand side, we see that $\operatorname{span}_{\mathbb{R}}\left\{e_{1}, e_{2}, e_{3}\right\} \subset T_{e_{0}} S^{7}$ is associative. We deduce the condition by calculating $* \tilde{\varphi}\left(e_{i}, e_{j}, e_{k}, \cdot\right)_{e_{l}}=0$ in the following cases:

(1) $c>0, A_{2}>0$,

(2) $c>0, A_{2}=0$,

(3) $c=0$.

Lemma 5.2. In the case $(1)$, the condition $* \tilde{\varphi}\left(e_{0}, e_{2}, e_{3}, \cdot\right)_{e_{1}}=0$ is equivalent to

(i) $s=0$

(ii) $s \neq 0, \quad A_{3}=B_{3}=0, \quad c^{2}-3 s^{2}=0, \quad$ or

(iii) $s \neq 0, \quad A_{3}=B_{3}=0, \quad c\left(A_{2}^{2}+3 A_{4}^{2}+3 B_{4}^{2}\right)-2 s A_{2} B_{4}=0$.

We abbreviate the case that (1) and (ii) hold as the case (1)-(ii) in the following.

Lemma 5.3. In the case (1)-(ii) or (1)-(iii), by normalizing $e_{2}$, we may assume that $A_{2}^{2}+A_{4}^{2}+B_{4}^{2}=1$. Then $* \tilde{\varphi}\left(e_{0}, e_{1}, e_{3}, \cdot\right)_{e_{2}}=0$ is equivalent to

(a) $A_{4}=B_{4}=0$,

(b) $A_{4}=0, \quad A_{2}^{2}-3 B_{4}^{2}=0$, or

(c) $A_{4}=0, \quad\left(c^{2}+3 s^{2}\right) A_{2}-2 c s B_{4}=0$.

Proof of Lemma 5.1. At $e_{0}$, we have

$$
\begin{aligned}
& \xi_{1}={ }^{t}(-i, 0,0,0), \\
& \xi_{2}={ }^{t}(0,-1,0,0), \\
& \xi_{3}={ }^{t}(0,-i, 0,0) .
\end{aligned}
$$

Setting $X_{0}={ }^{t}(0,0,1,0)$, we see $X_{0} \in \mathcal{H}_{e_{0}}$. Then $X_{i}=\Phi_{i}\left(X_{0}\right)$ for $i=1,2,3$ is described as

$$
\begin{aligned}
& X_{1}={ }^{t}(0,0, i, 0), \\
& X_{2}={ }^{t}(0,0,0,1), \\
& X_{3}={ }^{t}(0,0,0, i),
\end{aligned}
$$

and we have

$$
\begin{aligned}
& e_{1}=-c \xi_{1}+s X_{0}, \\
& e_{2}=-A_{2} \xi_{2}+A_{3} X_{0}+B_{3} X_{1}+A_{4} X_{2}+B_{4} X_{3} .
\end{aligned}
$$


By the definition of $\tilde{\varphi}$ in Proposition 4.3 we obtain

$$
\begin{aligned}
\tilde{\varphi}\left(e_{1}, e_{2}, \cdot\right)_{e_{0}}=\frac{27}{125} c\left(A_{2} \eta_{3}+\right. & \left.5 A_{3} X^{1}-5 B_{3} X^{0}+5 A_{4} X^{3}-5 B_{4} X^{2}\right) \\
+ & \frac{27}{25} s\left(-A_{2} X^{2}-B_{3} \eta_{1}-A_{4} \eta_{2}-B_{4} \eta_{3}\right) .
\end{aligned}
$$

Since $\tilde{g}=\frac{9}{25} \sum_{i=1}^{3} \eta_{i}+\frac{9}{5} \sum_{a=0}^{3} X^{a}$, we obtain the lemma.

Proof of Lemma 5.2. As in the proof of Lemma 5.1, we have at $e_{1}$

$$
\begin{aligned}
& \xi_{1}={ }^{t}(c, 0,-i s, 0), \\
& \xi_{2}={ }^{t}(0, i c, 0,-s), \\
& \xi_{3}={ }^{t}(0,-c, 0,-i s) .
\end{aligned}
$$

Setting $X_{0}={ }^{t}(0, i s, 0, c) \in \mathcal{H}_{e_{1}}, X_{i}=\Phi_{i}\left(X_{0}\right)$ for $i=1,2,3$ is described as

$$
\begin{aligned}
& X_{1}={ }^{t}(0,-s, 0, i c), \\
& X_{2}={ }^{t}(i s, 0,-c, 0), \\
& X_{3}={ }^{t}(-s, 0,-i c, 0) .
\end{aligned}
$$

Then by a direct computation, $* \tilde{\varphi}\left(e_{0}, e_{2}, e_{3},\right)_{e_{1}}=0$ is equivalent to

$$
\begin{array}{r}
4 s\left(c^{2}-3 s^{2}\right)\left(c A_{2}^{2}+3 c A_{4}^{2}+3 c B_{4}^{2}-2 s A_{2} B_{4}\right)=0, \\
s\left(\begin{array}{ccc}
c\left(-2 s^{2}+c^{2}\right) & -2 s^{3} & c\left(3 s^{2}+c^{2}\right) \\
3 s c & 3 s^{2}+c^{2} & -2 s c
\end{array}\right)\left(\begin{array}{l}
A_{3} A_{4} \\
A_{2} B_{3} \\
B_{3} B_{4}
\end{array}\right)=0, \\
s\left(\begin{array}{ccc}
s c & c^{2}-2 s^{2} & -\left(3 s^{2}+c^{2}\right) \\
c & -3 s & -2 s
\end{array}\right)\left(\begin{array}{l}
A_{2} A_{3} \\
A_{3} B_{4} \\
B_{3} A_{4}
\end{array}\right)=0 .
\end{array}
$$

It is clear that $s=0$ is a solution of (5.1), (5.2) and (5.3). We may assume that $s \neq 0$. From (5.2) and (5.3), we have

$$
\begin{aligned}
& \left(A_{3} A_{4}, A_{2} B_{3}, B_{3} B_{4}\right)=k\left(-\left(c^{2}+5 s^{2}\right), 5 s c, c^{2}\right), \\
& \left(A_{2} A_{3}, A_{3} B_{4}, B_{3} A_{4}\right)=l(5 s, c, c),
\end{aligned}
$$

for $k, l \in \mathbb{R}$. Since $A_{3} A_{4} B_{3} B_{4}=-k^{2} c^{2}\left(c^{2}+5 s^{2}\right)=l^{2} c^{2}$, we obtain $k=l=0$. The assumption $A_{2}>0$ gives $A_{3}=B_{3}=0$.

Proof of Lemma 5.3. As in the proof of Lemma 5.1, we have at $e_{1}$

$$
\begin{aligned}
& \xi_{1}={ }^{t}\left(0,-i A_{2}, 0, B_{4}-i A_{4}\right), \\
& \xi_{2}={ }^{t}\left(A_{2}, 0, A_{4}-i B_{4}, 0\right), \\
& \xi_{3}={ }^{t}\left(i A_{2}, 0, B_{4}+i A_{4}, 0\right) .
\end{aligned}
$$

Setting $X_{0}={ }^{t}\left(A_{4}+i B_{4}, 0,-A_{2}, 0\right) \in \mathcal{H}_{e_{2}}, X_{i}=\Phi_{i}\left(X_{0}\right)$ for $i=1,2,3$ is described as

$$
\begin{aligned}
& X_{1}={ }^{t}\left(-B_{4}+i A_{4}, 0,-i A_{2}, 0\right), \\
& X_{2}={ }^{t}\left(0, A_{4}-i B_{4}, 0,-A_{2}\right), \\
& X_{3}={ }^{t}\left(0, B_{4}+i A_{4}, 0,-i A_{2}\right) .
\end{aligned}
$$


Then by a direct computation, $* \tilde{\varphi}\left(e_{0}, e_{1}, e_{3}, \cdot\right)_{e_{2}}=0$ is equivalent to

$$
\begin{aligned}
& A_{4}\left\{c A_{2}\left(c A_{2}^{2}-2 s A_{2} B_{4}-3 c A_{4}^{2}-3 c B_{4}^{2}\right)\right. \\
&\left.+6 B_{4} s\left(-3 s A_{2} B_{4}+2 c A_{4}^{2}+2 c B_{4}^{2}\right)\right\}=0, \\
&\left(c^{2}+3 s^{2}\right) A_{2}^{3} B_{4}-2 c s A_{2}^{2} B_{4}^{2}+3\left(3 s^{2}-c^{2}\right) A_{2} A_{4}^{2} B_{4} \\
&-3\left(c^{2}+3 s^{2}\right) A_{2} B_{4}^{3}-6 c s A_{4}^{4}+6 c s B_{4}^{4}=0, \\
& s A_{2} A_{4}\left(c A_{2}^{2}-2 s A_{2} B_{4}+3 c A_{4}^{2}+3 c B_{4}^{2}\right)=0 .
\end{aligned}
$$

Suppose that $A_{4} \neq 0$ for a contradiction. Then (5.6) implies that

$$
c A_{2}^{2}-2 s A_{2} B_{4}+3 c A_{4}^{2}+3 c B_{4}^{2}=0 .
$$

Eliminating $A_{4}^{2}$ and $B_{4}^{2}$ from (5.4), we have $2 A_{2}\left(c A_{2}-5 s B_{4}\right)\left(c A_{2}+s B_{4}\right)=0$. However, the left hand side of (5.7) is greater than 0 when $B_{4}=\frac{c}{5 s} A_{2}$ or $-\frac{c}{s} A_{2}$. Thus we have $A_{4}=0$.

Then the left-hand sides of (5.4) and (5.6) vanish, and that of (5.5) is equal to $B_{4}\left(A_{2}^{2}-3 B_{4}^{2}\right)\left\{\left(c^{2}+3 s^{2}\right) A_{2}-2 c s B_{4}\right\}$, hence the proof is done.

Proof of Theorem 1.1. From Lemma 5.2 and 5.3 we consider the following cases:

Case (1)-(i) By the $\mathrm{Sp}(1)$-action, we may assume that $B_{3}=A_{4}=B_{4}=0$.

Normalizing $e_{2}$, we may assume $A_{2}^{2}+A_{3}^{2}=1$. Then as in the proof of Lemma 5.2 $* \tilde{\varphi}\left(e_{0}, e_{1}, e_{3}, \cdot\right)_{e_{2}}=0$ is equivalent to $A_{3}\left(A_{2}^{2}-A_{3}^{2}\right)=0$. Hence we have

$$
\begin{aligned}
\left(c, s, A_{2}, A_{3}, B_{3}, A_{4}, B_{4}\right)= & (1,0,1,0,0,0,0), \\
& \left(1,0, \frac{\sqrt{3}}{2}, \pm \frac{1}{2}, 0,0,0\right) .
\end{aligned}
$$

Case (1)-(ii)-(a) By normalizing $e_{2}$, we have $A_{2}=1$. Then we see

$$
\left(c, s, A_{2}, A_{3}, B_{3}, A_{4}, B_{4}\right)=\left(\frac{\sqrt{3}}{2}, \frac{1}{2}, 1,0,0,0,0\right) .
$$

In case (1)-(ii)-(b), (1)-(ii)-(c), and (1)-(iii)-(b), we have the following solutions:

$$
\begin{aligned}
\left(c, s, A_{2}, A_{3}, B_{3}, A_{4}, B_{4}\right)= & \left(\frac{\sqrt{3}}{2}, \frac{1}{2}, \frac{\sqrt{3}}{2}, 0,0,0, \pm \frac{1}{2}\right), \\
& \left(\frac{\sqrt{3}}{2}, \frac{1}{2}, \frac{1}{2}, 0,0,0, \frac{\sqrt{3}}{2}\right), \\
& \left(\frac{1}{2}, \frac{\sqrt{3}}{2}, \frac{\sqrt{3}}{2}, 0,0,0, \frac{1}{2}\right) .
\end{aligned}
$$

In case (1)-(iii)-(a) and (1)-(iii)-(c), we have no solutions.

The solution (5.8) corresponds to the $\mathbb{H}$-plane. The planes corresponding to (5.10), (5.11), (5.12), and (5.13) are congruent up to the $\mathrm{Sp}(1) \mathrm{Sp}(2)$-action to that of (5.9), which is not associative at $\left(e_{0}+e_{1}\right) / \sqrt{2}$ since $* \tilde{\varphi}\left(\left(-e_{0}+\right.\right.$ $\left.\left.e_{1}\right) / \sqrt{2},\left(e_{2}-e_{3}\right) / \sqrt{2},\left(e_{2}+e_{3}\right) / \sqrt{2}\right)_{\left(e_{0}+e_{1}\right) / \sqrt{2}} \neq 0$. 
Case (2) We may assume that the first and the second entries of $e_{3}$ are zero. Hence we have $B_{3} s=A_{4} s=B_{4} s=0$. If $s \neq 0$, we obtain the plane $V_{2}$. If $s=0$, the corresponding plane is congruent up to $\mathrm{Sp}(2)$-action to $V_{2}$.

Case (3) We may assume that the first and the second entries of $e_{2}$ and $e_{3}$ are zero. However, this implies that $e_{3}=0$, which is a contradiction.

\section{Classification of homogeneous associative sub- manifolds}

In this section, we prove Theorem 1.3. First, we classify compact Lie subgroups of $\operatorname{Sp}(1) \operatorname{Sp}(2)$ which have 3-dimensional orbits. Let $G$ be a compact connected Lie subgroup of $\operatorname{Sp}(1) \operatorname{Sp}(2)$. Suppose that $G$ has a 3 -dimensional orbit $A$. Since $G$ acts on $A$ as an isometry group, $\operatorname{dim} G \leq 3 \cdot(3+1) / 2=6$ and $\operatorname{dim} G \neq 5$. (see 13, Chapter IV, Theorem 9.1). We only have to consider the Lie algebra $\mathfrak{g} \subset \mathfrak{s p}(1) \oplus \mathfrak{s p}(2)$ of $G$.

\subsection{Case $\operatorname{dim} \mathfrak{g}=3$}

Suppose that $\operatorname{dim} \mathfrak{g}=3$. By the classification of the compact Lie algebras, $\mathfrak{g}$ is isomorphic to $\overline{\mathfrak{s u}(2) \text { or } \mathfrak{t}^{3}}$, where $\mathfrak{t}^{3}$ is a Lie algebra of the 3 -torus $T^{3}$. The case $\mathfrak{g}=\mathfrak{t}^{3}$ corresponds to the inclusion $T^{3} \hookrightarrow \mathrm{U}(1) \operatorname{Sp}(2) \subset \mathrm{U}(4)$ given by

$$
\left(e^{i \alpha}, e^{i \beta}, e^{i \gamma}\right) \mapsto \operatorname{diag}\left(e^{i(\alpha+\beta)}, e^{i(\alpha-\beta)}, e^{i(\alpha+\gamma)}, e^{i(\alpha-\gamma)}\right),
$$

which is a maximal torus of $\operatorname{Sp}(1) \operatorname{Sp}(2)$ and induces the $T^{3}$-action on $S^{7}$. Define the basis $\left\{F_{1}, F_{2}, F_{3}\right\}$ of the Lie algebra $\mathfrak{t}^{3} \cong \mathbb{R}^{3}$ of $T^{3}$ by

$$
F_{1}=(1,0,0), \quad F_{2}=(0,1,0), \quad F_{3}=(0,0,1) .
$$

Via the inclusion $\mathfrak{t}^{3} \hookrightarrow \mathfrak{u}(1) \oplus \mathfrak{s p}(2), F_{1}, F_{2}, F_{3}$ correspond to

$$
\left(\begin{array}{cccc}
i & & & \\
& i & & \\
& & i & \\
& & i
\end{array}\right),\left(\begin{array}{cccc}
i & & & \\
& -i & & \\
& & 0 & \\
& & & 0
\end{array}\right),\left(\begin{array}{cccc}
0 & & & \\
& 0 & & \\
& & i & \\
& & & -i
\end{array}\right) \text {, }
$$

respectively.

When $\mathfrak{g}=\mathfrak{s u}(2)$, we see that $\mathfrak{s u}(2)=\mathfrak{s p}(1)_{L}$ or $\mathfrak{s u}(2) \subset \mathfrak{s p}(2)_{R}$. Suppose that $\mathfrak{s u}(2) \subset \mathfrak{s p}(2)_{R}$. Recall that any representation of the compact Lie group $\mathrm{SU}(2)$ is completely reducible and the dimension of the real irreducible representation of $\mathrm{SU}(2)$ is of the form $4 k, 2 l-1(k, l \geq 1)$. Thus we have 3 types of inclusions $\mathfrak{s u}(2) \hookrightarrow \mathfrak{s o}(5)$ given by

$$
\begin{aligned}
& \mathfrak{s u}(2)=\mathfrak{s o}(3) \hookrightarrow \mathfrak{s o}(5), \\
& \mathfrak{s u}(2) \hookrightarrow \mathfrak{s o}(4) \hookrightarrow \mathfrak{s o}(5), \\
& \mathfrak{s u}(2) \hookrightarrow \mathfrak{s o}(5): \text { irreducibly. }
\end{aligned}
$$

The identification $\mathfrak{s p}(2)=\mathfrak{s o}(5)$ induces three types of inclusions $\mathrm{SU}(2) \hookrightarrow$ $\mathrm{Sp}(2)$. Hence we have the following four types of inclusions $\mathrm{SU}(2) \hookrightarrow \operatorname{Sp}(1) \operatorname{Sp}(2)$.

1. $\mathrm{SU}(2)=\mathrm{Sp}(1)_{L}$ acting on $S^{7}$ by (4.1), 
2. The inclusion $\mathrm{SU}(2) \hookrightarrow \mathrm{Sp}(2)$ given by

$$
\left(\begin{array}{cc}
a & -\bar{b} \\
b & \bar{a}
\end{array}\right) \mapsto\left(\begin{array}{cccc}
\bar{a} & & -b & \\
& a & & -\bar{b} \\
\bar{b} & & a & \\
& b & & \bar{a}
\end{array}\right)
$$

which induces the $\mathrm{SU}(2)$-action on $S^{7}$. Define the basis $\left\{E_{1}, E_{2}, E_{3}\right\}$ of the Lie algebra $\mathfrak{s u}(2)$ of $\mathrm{SU}(2)$ satisfying $\left[E_{i}, E_{i+1}\right]=2 E_{i+2}$ for $i \in \mathbb{Z} / 3$ by

$$
E_{1}=\left(\begin{array}{cc}
0 & 1 \\
-1 & 0
\end{array}\right), \quad E_{2}=\left(\begin{array}{cc}
0 & i \\
i & 0
\end{array}\right), \quad E_{3}=\left(\begin{array}{cc}
i & 0 \\
0 & i
\end{array}\right)
$$

Via this inclusion $\mathfrak{s u}(2) \hookrightarrow \mathfrak{s p}(2), E_{1}, E_{2}, E_{3}$ correspond to

$$
\left(\begin{array}{llll} 
& & 1 & \\
-1 & & & 1 \\
& -1 & &
\end{array}\right),\left(\begin{array}{llll} 
& & -i & \\
& & & i \\
-i & & & \\
& i & &
\end{array}\right),\left(\begin{array}{llll}
-i & & \\
& i & & \\
& & i & \\
& & & -i
\end{array}\right)
$$

respectively.

3. The inclusion $\mathrm{SU}(2) \hookrightarrow \mathrm{Sp}(2)$ given by

$$
A \mapsto\left(\begin{array}{cc}
A & O_{2} \\
O_{2} & I_{2}
\end{array}\right)
$$

Via this inclusion $\mathfrak{s u}(2) \hookrightarrow \mathfrak{s p}(2), E_{1}, E_{2}, E_{3}$ correspond to

$$
\left(\begin{array}{ccc} 
& 1 & \\
-1 & & \\
& & O_{2}
\end{array}\right),\left(\begin{array}{ccc} 
& i & \\
i & & \\
& & O_{2}
\end{array}\right),\left(\begin{array}{lll}
i & & \\
& -i & \\
& & O_{2}
\end{array}\right) \text {, }
$$

respectively.

4. The inclusion $\mathrm{SU}(2) \hookrightarrow \mathrm{Sp}(2)$ given by

$$
\left(\begin{array}{cc}
a & -\bar{b} \\
b & \bar{a}
\end{array}\right) \mapsto\left(\begin{array}{cccc}
a^{3} & -\bar{b}^{3} & \sqrt{3} a \bar{b}^{2} & -\sqrt{3} a^{2} \bar{b} \\
b^{3} & \bar{a}^{3} & \sqrt{3} \bar{a}^{2} b & \sqrt{3} \bar{a} b^{2} \\
\sqrt{3} a b^{2} & -\sqrt{3} \bar{a}^{2} \bar{b} & \bar{a}\left(|a|^{2}-2|b|^{2}\right) & b\left(2|a|^{2}-|b|^{2}\right) \\
\sqrt{3} a^{2} b & \sqrt{3} \bar{a} \bar{b}^{2} & -\bar{b}\left(2|a|^{2}-|b|^{2}\right) & a\left(|a|^{2}-2|b|^{2}\right)
\end{array}\right),
$$

which induces the $\mathrm{SU}(2)$-action on $S^{7}$. This action is an irreducible representation of $\mathrm{SU}(2)$ on $\mathbb{C}^{4}$. This is the induced action of $\mathrm{SU}(2)$ on $V_{3}=\mathbb{C}^{4}$ from the standard action on $\mathbb{C}^{2}$, where we use the notation of Lemma 7.6. Via $\mathfrak{s u}(2) \hookrightarrow \mathfrak{s p}(2), E_{1}, E_{2}, E_{3}$ correspond to

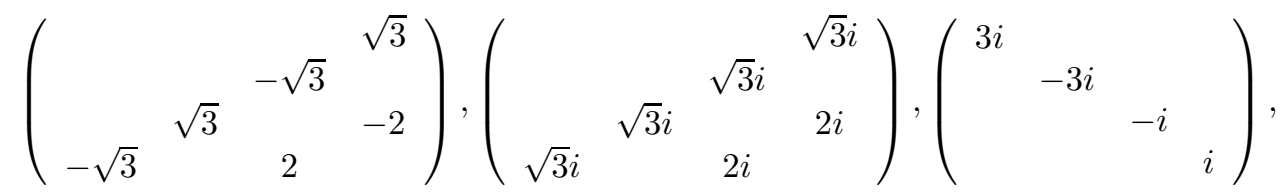

respectively. 


\subsection{Case $\operatorname{dim} \mathfrak{g}=4$}

By the classification of the compact Lie algebras, $\mathfrak{g}$ is isomorphic to $\mathfrak{s u}(2) \oplus \mathbb{R}$. Since the inclusions $\mathfrak{s u}(2) \hookrightarrow \mathfrak{s p}(1) \oplus \mathfrak{s p}(2)$ are classified, we have to find the 1-dimensional Lie subalgebras which commute with $\mathfrak{s u}(2)$. Set

$$
Z(\mathfrak{s u}(2))=\{X \in \mathfrak{s p}(1) \oplus \mathfrak{s p}(2) ;[X, Y]=0 \text { for any } Y \in \mathfrak{s u}(2)\}
$$

First consider the case $\mathfrak{s u}(2)=\mathfrak{s p}(1)_{L}$. Then we have $Z(\mathfrak{s u}(2))=\mathfrak{s p}(2)_{R}$. Take any 1-dimensional subspace $\mathfrak{k} \subset \mathfrak{s p}(2)_{R}$ and suppose that $G$ is the Lie subgroup of $\operatorname{Sp}(1) \operatorname{Sp}(2)$ whose Lie algebra is $\mathfrak{s u}(2) \oplus \mathfrak{k}$. Since the $\operatorname{Sp}(1)_{L}$-action commutes with the $\operatorname{Sp}(2)_{R}$-action, the $G$-orbit through $p \in S^{7}$ should be contained in $\operatorname{Sp}(1) \cdot p$ so that it is 3 -dimensional. Thus this case is reduced to that of (4.1).

Next, suppose that $\mathfrak{s u}(2) \subset \mathfrak{s p}(2)$ is induced from (6.4). In this case, we have $Z(\mathfrak{s u}(2))=\mathfrak{s p}(1)_{L} \oplus(\mathbb{R} \operatorname{diag}(i,-i, i,-i))_{R}$. The Lie subgroup $G \subset \operatorname{Sp}(2)$ whose Lie algebra is $(\mathfrak{s u}(2) \oplus \mathbb{R} \operatorname{diag}(i,-i, i,-i))_{R}$ is $\mathrm{U}(2)$ whose restriction to $\mathrm{SU}(2)$ is given by (6.4). This $\mathrm{U}(2)$ action has the same orbits as the $\mathrm{SU}(2)$-action. The new 3 -dimensional orbits do not appear from $\mathfrak{s p}(1)_{L}$, and this case is reduced to that of (6.4).

Suppose that $\mathfrak{s u}(2) \subset \mathfrak{s p}(2)$ is induced from (6.7). In this case, we have $Z(\mathfrak{s u}(2))=\mathfrak{s p}(1)_{L} \oplus\left(\begin{array}{cc}\mathrm{O}_{2} & \\ & \mathfrak{s u}(2)\end{array}\right)_{R}$. This case is also reduced to that of (6.7) in the same way.

Suppose that $\mathfrak{s u}(2) \subset \mathfrak{s p}(2)$ is induced from (6.9). In this case, we have $Z(\mathfrak{s u}(2))=\mathfrak{s p}(1)_{L}$. This case is also reduced to that of (6.9) in the same way.

\subsection{Case $\operatorname{dim} \mathfrak{g}=6$}

By the classification of the compact Lie algebras, $\mathfrak{g}$ is isomorphic to $\mathfrak{s u}(2) \oplus \mathfrak{t}^{3}$ or $\mathfrak{s u}(2) \oplus \mathfrak{s u}(2)$. When $\mathfrak{g} \cong \mathfrak{s u}(2) \oplus \mathfrak{t}^{3}$, we have $\mathfrak{g} \cong \mathfrak{t}_{L}^{1} \oplus\left(\mathfrak{s u}(2) \oplus \mathfrak{t}^{2}\right)_{R}$. Since there are no 2-dimensional commutative Lie subalgebras of $\mathfrak{s p}(2)$ which commute with $\mathfrak{s u}(2)$ by Section 6.2 this case does not occur.

$$
\text { When } \mathfrak{g} \cong \mathfrak{s u}(2) \oplus \mathfrak{s u}(2), \text { we have } G=\mathrm{Sp}(1)_{L} \cdot \mathrm{SU}(2)_{R} \text { or }\left(\begin{array}{ll}
\mathrm{SU}(2) & \\
& \mathrm{SU}(2)
\end{array}\right)_{R},
$$
which reduces to the case above.

Thus we only have to consider the orbits of (6.1), (4.1), (6.4), (6.7), and (6.9).

\section{$6.4 T^{3}$-orbits}

We classify associative submanifolds which are orbits of $T^{3}$ acting on $S^{7}$ as (6.1).

Proposition 6.1. Up to the $\operatorname{Sp}(1) \operatorname{Sp}(2)$-action, $T^{3} \cdot \frac{1}{2}^{t}(1,1,1, i)$ is the unique associative submanifold in the squashed $S^{7}$ which is an orbit of the $T^{3}$-action.

Remark 6.2. The associative orbit $A_{1}=T^{3} \cdot \frac{1}{2}^{t}(1,1,1, i)$ is the Hopf lift of a $I_{1}^{\prime}$-holomorphic curve in $\mathbb{C} P^{3}$, where $I_{1}^{\prime}$ is defined by (4.2). We have

$$
A_{1}=\left\{{ }^{t}\left(z_{1}, z_{2}, z_{3}, z_{4}\right) \in S^{7} ; \quad \begin{array}{c}
\left|z_{1}\right|=\left|z_{2}\right|=\left|z_{3}\right|=\left|z_{4}\right| \\
\operatorname{Re}\left(z_{1} z_{2} \bar{z}_{3} \bar{z}_{4}\right)=0, \operatorname{Im}\left(z_{1} z_{2} \bar{z}_{3} \bar{z}_{4}\right)<0
\end{array}\right\}
$$


which is a special Legendrian given in [5] via ${ }^{t}\left(z_{1}, z_{2}, z_{3}, z_{4}\right) \mapsto{ }^{t}\left(z_{1}, z_{2}, \bar{z}_{3}, \bar{z}_{4}\right)$. The inclusion (6.1) induces the metric $\frac{3}{5}\left(F^{1}\right)^{2}+\frac{27}{50}\left(F^{2}\right)^{2}+\frac{27}{50}\left(F^{3}\right)^{2}$, where $\left\{F^{i}\right\}$ is the dual of $\left\{F_{i}\right\}$.

Proof. Fix $p_{0}={ }^{t}\left(z_{1}, z_{2}, z_{3}, z_{4}\right) \in S^{7}$ and set $A=T^{3} \cdot p_{0}$. Then the tangent space $T_{p_{0}} A$ is spanned by the vectors $F_{i}^{*}$ generated by $F_{i}$ in (6.2):

$$
\begin{aligned}
& \left(F_{1}^{*}\right)_{p_{0}}=i^{t}\left(z_{1}, z_{2}, z_{3}, z_{4}\right)=-\xi_{1}, \\
& \left(F_{2}^{*}\right)_{p_{0}}=i^{t}\left(z_{1},-z_{2}, 0,0\right) \\
& \left(F_{3}^{*}\right)_{p_{0}}=i^{t}\left(0,0, z_{3},-z_{4}\right) .
\end{aligned}
$$

By Lemma 2.6, we consider the condition $\left.* \tilde{\varphi}\left(F_{1}^{*}, F_{2}^{*}, F_{3}^{*}, \cdot\right)\right|_{T_{p_{0}} S^{7}}=0$. We easily see that $-i\left(F_{1}^{*}\right) * \tilde{\varphi}=\left(3^{4} / 5^{3}\right) \operatorname{Im}\left(\left(\eta_{2}-i \eta_{3}\right) \wedge d\left(\eta_{2}+i \eta_{3}\right)\right)$. From Lemma 4.1, we have

$$
\begin{aligned}
\left(\eta_{2}+i \eta_{3}\right)\left(F_{2}^{*}\right) & =2 i z_{1} z_{2}, & \left(\eta_{2}+i \eta_{3}\right)\left(F_{3}^{*}\right) & =2 i z_{3} z_{4}, \\
d\left(\eta_{2}+i \eta_{3}\right)\left(F_{2}^{*}, \cdot\right) & =-2 i d\left(z_{1} z_{2}\right), & d\left(\eta_{2}+i \eta_{3}\right)\left(F_{3}^{*}, \cdot\right) & =-2 i d\left(z_{3} z_{4}\right),
\end{aligned}
$$

which implies that the condition $\left.* \tilde{\varphi}\left(F_{1}^{*}, F_{2}^{*}, F_{3}^{*}, \cdot\right)\right|_{T_{p_{0}} S^{7}}=0$ is equivalent to $d\left(\operatorname{Im}\left(z_{1} z_{2} \bar{z}_{3} \bar{z}_{4}\right)\right)=0$. The restriction of this form to $T S^{7}$ is given by $d\left(\operatorname{Im}\left(z_{1} z_{2} \bar{z}_{3} \bar{z}_{4}\right)\right)-$ $d\left(\operatorname{Im}\left(z_{1} z_{2} \bar{z}_{3} \bar{z}_{4}\right)\right)\left(r \frac{\partial}{\partial r}\right) \frac{d r}{r}=\operatorname{Re}\left(\sum_{j=1}^{4} \zeta_{j} d z_{j}\right)$, where $r \frac{\partial}{\partial r}$ is a position vector, $\frac{d r}{r}$ is its dual, and

$$
\left(\begin{array}{l}
\zeta_{1} \\
\zeta_{2} \\
\zeta_{3} \\
\zeta_{4}
\end{array}\right)=\left(\begin{array}{c}
-i z_{2} \bar{z}_{3} \bar{z}_{4} \\
-i z_{1} \bar{z}_{3} \bar{z}_{4} \\
i \bar{z}_{1} \bar{z}_{2} z_{4} \\
i \bar{z}_{1} \bar{z}_{2} z_{3}
\end{array}\right)-4 \operatorname{Im}\left(z_{1} z_{2} \bar{z}_{3} \bar{z}_{4}\right)\left(\begin{array}{c}
\bar{z}_{1} \\
\bar{z}_{2} \\
\bar{z}_{3} \\
\bar{z}_{4}
\end{array}\right) .
$$

Thus we see that the condition $\left.* \tilde{\varphi}\left(F_{1}^{*}, F_{2}^{*}, F_{3}^{*}, \cdot\right)\right|_{T_{p_{0}} S^{7}}=0$ is equivalent to $\zeta_{j}\left(p_{0}\right)=0$ for $j=1, \cdots, 4$. On the other hand, setting

$$
\Sigma=\left\{{ }^{t}\left(x_{1}, x_{2}, x_{3}, x_{4}+i y_{4}\right) \in S^{7} \subset \mathbb{C}^{4} ; x_{j}, y_{4} \in \mathbb{R}, x_{1}, x_{2}, x_{3} \geq 0\right\},
$$

we have $S^{7}=T^{3} \cdot \Sigma$. Hence we may assume that $p_{0} \in \Sigma$ and $x_{1}, x_{2}, x_{3} \neq 0$ so that $T^{3} \cdot p_{0}$ is 3 -dimensional. Then we can solve $\zeta_{j}=0$ easily to obtain

$$
x_{1}=x_{2}=x_{3}=1 / 2, \quad x_{4}=0, \quad y_{4}= \pm 1 / 2 .
$$

The $T^{3}$-orbit through ${ }^{t}(1,1,1, i) / 2$ is mapped to that through ${ }^{t}(1,1,1,-i) / 2$ by $\left(\begin{array}{cc}I_{2} & 0 \\ 0 & K\end{array}\right) \in \operatorname{Sp}(2)$, where $K=\left(\begin{array}{cc}0 & -i \\ -i & 0\end{array}\right)$, and we obtain the statement.

\section{5 $\mathrm{SU}(2)$-orbits}

We consider the SU(2)-orbits of (4.1), (6.4), (6.7), or (6.9). First, we introduce a useful lemma to study associative orbits.

Lemma 6.3. ([9] Lemma 5.6.) Let $(V, \rho)$ be an orthogonal representation of $\mathrm{SU}(2),\langle\cdot, \cdot\rangle$ be an $\mathrm{SU}(2)$-invariant inner product on $V$, and $S_{1} \subset V$ be the unit 
sphere. Let $M=\mathrm{SU}(2) \cdot p$ be a 3-dimensional orbit through $p \in S_{1}$. Define the function $\lambda_{j}: M \rightarrow \mathbb{R}$ for $j=1,2,3$ by

$$
\lambda_{j}=\left.\left\langle\left(\rho_{*}\left(E_{j}\right)\right)^{*},\left(\rho_{*}\left(E_{j}\right)\right)^{*}\right\rangle\right|_{M},
$$

where $\left\{E_{j}\right\}$ is a basis of $\mathrm{su}(2)$ satisfying $\left[E_{i}, E_{i+1}\right]=2 E_{i+2}$ for $i \in \mathbb{Z} / 3$ and $\left(\rho_{*}\left(E_{j}\right)\right)^{*}$ is a vector field on $V$ generated by $\rho_{*}\left(E_{j}\right) \in \mathfrak{g l}(V)$. Denote by $\left\{E^{j}\right\}$ the dual 1-form on $M$ of $\left\{\left.\left(\rho_{*}\left(E_{j}\right)\right)^{*}\right|_{M}\right\}$. Then there exists $g \in \mathrm{SU}(2)$, the induced metric $\left.\langle\cdot, \cdot\rangle\right|_{M}$ is described as

$$
\left.\langle\cdot, \cdot\rangle\right|_{M}=\sum_{j=1}^{3} \lambda_{j}\left(E^{j}\right)^{2}
$$

at $g \cdot p \in M$. Moreover, $\left(M,\left.\langle\cdot, \cdot\rangle\right|_{M}\right)$ is a space of constant curvature $k$ if and only if $\lambda_{1}=\lambda_{2}=\lambda_{3}=1 / k$.

Remark 6.4. (9] Remark 5.4.) There exists $g^{\prime} \in \mathrm{SU}(2)$ satisfying (6.11) and $\lambda_{1}\left(g^{\prime}\right)=\lambda_{a}(g), \lambda_{2}\left(g^{\prime}\right)=\lambda_{b}(g), \lambda_{3}\left(g^{\prime}\right)=\lambda_{c}(g)$, where $\{a, b, c\}$ is any permutation of $\{1,2,3\}$. Thus we can "permute" the $\lambda_{j}$.

\subsubsection{SU(2)-orbits 1}

If an SU(2)-action is given by (4.1), the orbit is the intersection of a quaternionic plane and $S^{7}$, which is an obvious totally geodesic associative submanifold.

\subsection{2 $\mathrm{SU}(2)$-orbits 2}

Consider the $\mathrm{SU}(2)$-action given by (6.4). Let $A$ be an $\mathrm{SU}(2)$-orbit through $p_{0}={ }^{t}\left(z_{1}, z_{2}, z_{3}, z_{4}\right)$. Then the tangent space to $A$ at $p_{0}$ is spanned by the vectors $E_{i}^{*}$ generated by $E_{i}$ in 6.5 :

$$
\begin{aligned}
& \left(E_{1}^{*}\right)_{p_{0}}={ }^{t}\left(z_{3}, z_{4},-z_{1},-z_{2}\right), \\
& \left(E_{2}^{*}\right)_{p_{0}}=i^{t}\left(-z_{3}, z_{4},-z_{1}, z_{2}\right), \\
& \left(E_{3}^{*}\right)_{p_{0}}=i^{t}\left(-z_{1}, z_{2}, z_{3},-z_{4}\right) .
\end{aligned}
$$

We easily see that $g\left(E_{i}^{*}, E_{j}^{*}\right)_{p_{0}}=\delta_{i j}$, where $g$ is the standard metric on $S^{7}$. Then from Lemma 6.3. $A$ is a constant curvature 1 submanifold of $\left(S^{7}, g\right)$. Thus $A$ is of the form $V \cap S^{7}$, where $V \subset \mathbb{R}^{8}$ is a 4-plane. These associative submanifolds are classified by Theorem 1.1 .

\subsection{3 $\mathrm{SU}(2)$-orbits 3}

Consider the $\mathrm{SU}(2)$-action given by (6.7). Let $A$ be an $\mathrm{SU}(2)$-orbit through $p_{0}=$ ${ }^{t}\left(z_{1}, z_{2}, z_{3}, z_{4}\right)$. By the $\mathrm{SU}(2)$-action, we may assume that $p_{0}={ }^{t}\left(x_{1}, 0, z_{3}, z_{4}\right)$ where $x_{1}>0, z_{3}, z_{4} \in \mathbb{C}$. Then the tangent space to $A$ at $p_{0}$ is spanned by the vectors $E_{i}^{*}$ generated by $E_{i}$ in $(\underline{6.5})$ :

$$
\begin{aligned}
& \left(E_{1}^{*}\right)_{p_{0}}={ }^{t}\left(0,-x_{1}, 0,0\right), \\
& \left(E_{2}^{*}\right)_{p_{0}}={ }^{t}\left(0, i x_{1}, 0,0\right), \\
& \left(E_{3}^{*}\right)_{p_{0}}={ }^{t}\left(i x_{1}, 0,0,0\right) .
\end{aligned}
$$


We compute

$$
\begin{gathered}
\left(\eta_{i}\left(E_{j}^{*}\right)\right)=\left(\begin{array}{ccc}
0 & 0 & -x_{1}^{2} \\
-x_{1}^{2} & 0 & 0 \\
0 & -x_{1}^{2} & 0
\end{array}\right), \\
\left(\begin{array}{c}
d \eta_{j}\left(E_{1}^{*}, E_{2}^{*}\right) \\
d \eta_{j}\left(E_{1}^{*}, E_{3}^{*}\right) \\
d \eta_{j}\left(E_{2}^{*}, E_{3}^{*}\right)
\end{array}\right)=\left(\begin{array}{ccc}
x_{1}^{2} & 0 & 0 \\
0 & 0 & -x_{1}^{2} \\
0 & -x_{1}^{2} & 0
\end{array}\right), \\
\left(i\left(E_{i}^{*}\right) d \eta_{1}\right)=2 x_{1}\left(\begin{array}{c}
\operatorname{Im}\left(d z_{2}\right) \\
\operatorname{Re}\left(d z_{2}\right) \\
\operatorname{Re}\left(d z_{1}\right)
\end{array}\right), \quad\left(i\left(E_{i}^{*}\right) d\left(\eta_{2}+i \eta_{3}\right)\right)=2 x_{1}\left(\begin{array}{c}
-d z_{1} \\
i d z_{1} \\
-i d z_{2}
\end{array}\right), \\
\sum_{i=1}^{3} d \eta_{i}\left(E_{1}^{*}, E_{2}^{*}, E_{3}^{*}, \cdot\right)=12 x_{1}^{3} d x_{1}, \quad d\left(\eta_{123}\right)=2 x_{1}^{5} d x_{1} .
\end{gathered}
$$

Since $* \tilde{\varphi}=\frac{27}{25}\left(\frac{1}{8} \sum_{i=1}^{3}\left(d \eta_{i}\right)^{2}+\frac{4}{5} d\left(\eta_{123}\right)\right)$, we obtain $* \tilde{\varphi}\left(E_{1}^{*}, E_{2}^{*}, E_{3}^{*}, \cdot\right)=\frac{5}{54} x_{1}^{3}(15+$ $\left.16 x_{1}^{2}\right) d x_{1}$. The restriction of $d x_{1}$ to $T S^{7}$ is given by

$$
d x_{1}-d x_{1}\left(r \frac{\partial}{\partial r}\right) \frac{d r}{r}=d x_{1}-x_{1}\left(x_{1} d x_{1}+\operatorname{Re}\left(z_{3} d z_{3}+z_{4} d z_{4}\right)\right),
$$

where $r \frac{\partial}{\partial r}$ is a position vector and $\frac{d r}{r}$ is its dual. This implies that $\left.* \tilde{\varphi}\left(E_{1}, E_{2}, E_{3}, \cdot\right)\right|_{T_{p_{0}} S^{7}}=$ 0 is equivalent to $x_{1}=1, z_{3}=z_{4}=0$, and the resulting associative submanifold is $\left\{\left(z_{1}, z_{2}, 0,0\right) \in \mathbb{C}^{4} ;\left|z_{1}\right|^{2}+\left|z_{2}\right|^{2}=1\right\}$.

\subsection{4 $\mathrm{SU}(2)$-orbits 4}

For the $\mathrm{SU}(2)$-action given by (6.9), we obtain the following.

Proposition 6.5. Let $A$ be an associative submanifold in the squashed $S^{7}$ which is an orbit of the $\mathrm{SU}(2)$-action given in (6.9). Then up to the $\mathrm{Sp}(1) \mathrm{Sp}(2)$-action,

$$
A=A_{2}:=\mathrm{SU}(2) \cdot{ }^{t}(1,0,0,0) \quad \text { or } \quad A_{3}:=\mathrm{SU}(2) \cdot{ }^{t}(0,0,1,0) .
$$

Remark 6.6. The associative orbit $A_{2}$ is the Hopf lift of a horizontal holomorphic curve

$$
\left\{\left[a^{3}: b^{3}: \sqrt{3} a b^{2}: \sqrt{3} a^{2} b\right] \in \mathbb{C} P^{3} ; a, b \in \mathbb{C},|a|^{2}+|b|^{2}=1\right\}
$$

in $\mathbb{C} P^{3}$. This is a degree $3 \mathbb{C} P^{1}$ in $\mathbb{C} P^{3}$ of the constant curvature called the Veronese curve. The associative orbit $A_{3}$ is the Hopf lift of a null-torsion $I_{1}^{\prime}$ holomorphic curve in $\mathbb{C} P^{3}$, which is defined in Definition 7.15. The inclusion (6.9) induces $\left.\tilde{g}\right|_{A_{2}}=\frac{27}{25}\left(5\left(E^{1}\right)^{2}+5\left(E^{2}\right)^{2}+3\left(E^{3}\right)^{2}\right)$ and $\left.\tilde{g}\right|_{A_{3}}=\frac{9}{25}\left(19\left(E^{1}\right)^{2}+\right.$ $\left.19\left(E^{2}\right)^{2}+\left(E^{3}\right)^{2}\right)$, where we use the notation of Lemma 6.3.

Remark 6.7. Set $A_{2}(a, b):=\mathrm{SU}(2) \cdot{ }^{t}(a, b, 0,0)$ and $A_{3}(a, b):=\mathrm{SU}(2) \cdot{ }^{t}(0,0, a, b)$ for $a, b \in \mathbb{C},|a|^{2}+|b|^{2}=1$. Then by the action of $a+b j \in \mathrm{Sp}(1)_{L}, A_{j}$ is congruent to $A_{j}(a, b)(j=2,3)$. Via ${ }^{t}\left(z_{1}, z_{2}, z_{3}, z_{4}\right) \mapsto^{t}\left(z_{1}, z_{4}, z_{3}, z_{2}\right), A_{2}\left(\frac{1}{\sqrt{2}}, \frac{1}{\sqrt{2}}\right)$ is special Legendrian given by 8$]$. 
Proof of Proposition 6.5. Let $A$ be an $\mathrm{SU}(2)$-orbit through $p_{0}={ }^{t}\left(z_{1}, z_{2}, z_{3}, z_{4}\right)$. Then the tangent space to $A$ at $p_{0}$ is spanned by the vectors $E_{i}^{*}$ generated by $E_{i}$ in (6.5):

$$
\begin{aligned}
& \left(E_{1}^{*}\right)_{p_{0}}={ }^{t}\left(\sqrt{3} z_{4},-\sqrt{3} z_{3}, \sqrt{3} z_{2}-2 z_{4},-\sqrt{3} z_{1}+2 z_{3}\right), \\
& \left(E_{2}^{*}\right)_{p_{0}}={ }^{t}\left(\sqrt{3} i z_{4}, \sqrt{3} i z_{3}, \sqrt{3} i z_{2}+2 i z_{4}, \sqrt{3} i z_{1}+2 i z_{3}\right), \\
& \left(E_{3}^{*}\right)_{p_{0}}={ }^{t}\left(3 i z_{1},-3 i z_{2},-i z_{3}, i z_{4}\right) .
\end{aligned}
$$

Since $\mathrm{SU}(2) \subset \mathrm{Sp}(2)$-action preserves $\eta_{j}$, we have $L_{E_{j}^{*}} \eta_{i}=d \eta_{i}\left(E_{j}^{*}, \cdot\right)+d\left(\eta_{i}\left(E_{j}^{*}\right)\right)=$ 0 . Then by the equation $\left[E_{j}^{*}, E_{j+1}^{*}\right]=-2 E_{j+2}^{*}$ for $j \in \mathbb{Z} / 3$, we have

$$
\begin{aligned}
\sum_{i=1}^{3}\left(d \eta_{i}\right)^{2}\left(E_{1}^{*}, E_{2}^{*}, E_{3}^{*}, \cdot\right) & =2 \sum_{i, j=1}^{3} d\left(\eta_{i}\left(E_{j}^{*}\right)^{2}\right), \\
d\left(\eta_{123}\right)\left(E_{1}^{*}, E_{2}^{*}, E_{3}^{*}, \cdot\right) & =-d\left(\eta_{123}\left(E_{1}^{*}, E_{2}^{*}, E_{3}^{*}\right)\right) .
\end{aligned}
$$

We compute

$$
\begin{aligned}
\eta_{1}\left(E_{2}^{*}\right)+i \eta_{1}\left(E_{1}^{*}\right) & =-2 \sqrt{3}\left(\bar{z}_{1} z_{4}+z_{2} \bar{z}_{3}\right)-4 z_{3} \bar{z}_{4}, \\
\eta_{1}\left(E_{3}^{*}\right) & =-3\left|z_{1}\right|^{2}+3\left|z_{2}\right|^{2}+\left|z_{3}\right|^{2}-\left|z_{4}\right|^{2}, \\
\left(\eta_{2}+i \eta_{3}\right)\left(E_{1}^{*}\right) & =2 \sqrt{3}\left(z_{1} z_{3}+z_{2} z_{4}\right)-2\left(z_{3}^{2}+z_{4}^{2}\right), \\
\left(\eta_{2}+i \eta_{3}\right)\left(E_{2}^{*}\right) & =2 \sqrt{3} i\left(-z_{1} z_{3}+z_{2} z_{4}\right)+2 i\left(-z_{3}^{2}+z_{4}^{2}\right), \\
\left(\eta_{2}+i \eta_{3}\right)\left(E_{3}^{*}\right) & =6 i z_{1} z_{2}-2 i z_{3} z_{4},
\end{aligned}
$$

Then we have $\sum_{i, j=1}^{3} \eta_{i}\left(E_{j}^{*}\right)^{2}=9$ and $\sum_{i=1}^{3}\left(d \eta_{i}\right)^{2}\left(E_{1}^{*}, E_{2}^{*}, E_{3}^{*}, \cdot\right)=0$ by (6.12). Since $* \tilde{\varphi}=\frac{27}{25}\left(\frac{1}{8} \sum_{i=1}^{3}\left(d \eta_{i}\right)^{2}+\frac{4}{5} d\left(\eta_{123}\right)\right)$, the condition $* \tilde{\varphi}\left(E_{1}^{*}, E_{2}^{*}, E_{3}^{*}, \cdot\right)=0$ is equivalent to

$$
d(\operatorname{det} M)=0,
$$

where $M=\left(\eta_{i}\left(E_{j}^{*}\right)\right)$

Now, we use Lemma 6.3. We may assume that $\left\{E_{1}^{*}, E_{2}^{*}, E_{3}^{*}\right\}$ are mutually orthogonal at $p_{0}={ }^{t}\left(z_{1}, z_{2}, z_{3}, z_{4}\right)$ with respect to $g$. Then we have

$$
z_{1} \bar{z}_{4}-\bar{z}_{2} z_{3}=0, \quad \operatorname{Im}\left(z_{1} \bar{z}_{3}+\bar{z}_{2} z_{4}\right)=0 .
$$

Setting

$$
\begin{aligned}
& \lambda_{1}=\left|E_{1}^{*}\right|^{2}=4\left(\left|z_{3}\right|^{2}+\left|z_{4}\right|^{2}\right)-4 \sqrt{3} \operatorname{Re}\left(z_{1} \bar{z}_{3}+\bar{z}_{2} z_{4}\right)+3, \\
& \lambda_{2}=\left|E_{2}^{*}\right|^{2}=4\left(\left|z_{3}\right|^{2}+\left|z_{4}\right|^{2}\right)+4 \sqrt{3} \operatorname{Re}\left(z_{1} \bar{z}_{3}+\bar{z}_{2} z_{4}\right)+3, \\
& \lambda_{3}=\left|E_{3}^{*}\right|^{2}=8\left(\left|z_{1}\right|^{2}+\left|z_{2}\right|^{2}\right)+1 .
\end{aligned}
$$

We consider the following two cases as the proof of Lemma 5.7 in [7]:

(1) all of the $\lambda_{j}$ are distinct, (2) at least two of the $\lambda_{j}$ are equal.

Consider the case (1). Since we can permute the $\lambda_{j}$ by Remark 6.4 we may assume that $\lambda_{3}<\lambda_{1}<\lambda_{2}$. The inequality $\lambda_{1}<\lambda_{2}$ implies that $\operatorname{Re}\left(z_{1} \bar{z}_{3}+\right.$ 
$\left.z_{2} \bar{z}_{4}\right)>0$. Thus we have $\left(z_{1}, z_{2}\right),\left(z_{3}, z_{4}\right) \neq 0$. From (6.13), there exists $\mu \in \mathbb{R}$ satisfying

$$
z_{3}=\mu z_{1}, \quad z_{4}=\mu z_{2} .
$$

Note that $\lambda_{3}<\lambda_{1}$ is equivalent to $\mu>\sqrt{3}$. Moreover, since the $\operatorname{Sp}(1)_{L}$-action

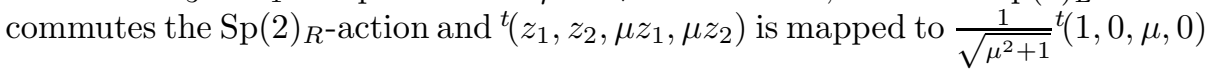
by $\left(\bar{z}_{1}-z_{2} j\right) / \sqrt{\left|z_{1}\right|^{2}+\left|z_{2}\right|^{2}} \in \operatorname{Sp}(1)_{L}$, we may assume that $p_{0}={\frac{1}{{\sqrt{\mu^{2}+1}}^{t}}}^{t}(1,0, \mu, 0)$.

Set $v={ }^{t}(-\mu, 0,1,0) \in T_{p_{0}} S^{7}$. Then we compute

$$
\begin{aligned}
M_{p_{0}} & =\frac{1}{\mu^{2}+1}\left(\begin{array}{ccc}
0 & 0 & \mu^{2}-3 \\
2 \mu(-\mu+\sqrt{3}) & 0 & 0 \\
0 & -2 \mu(\mu+\sqrt{3}) & 0
\end{array}\right), \\
(v(M))_{p_{0}} & =\frac{1}{\sqrt{\mu^{2}+1}}\left(\begin{array}{ccc}
0 & 0 & 8 \mu \\
-2(\sqrt{3} \mu-1)(\mu+\sqrt{3}) & 0 & 0 \\
0 & 2(\sqrt{3} \mu+1)(\mu-\sqrt{3}) & 0
\end{array}\right),
\end{aligned}
$$

where $v(M)$ is the derivative of $M$ with respect to $v$. Then we have

$$
\begin{aligned}
d(\operatorname{det} M)_{p_{0}}(v) & =\operatorname{det} M_{p_{0}} \cdot \operatorname{tr}\left(v(M) M^{-1}\right)_{p_{0}} \\
& =24 \mu\left(\mu^{2}-3\right)\left(3 \mu^{2}-1\right)\left(\mu^{2}+1\right)^{-5 / 2}>0 .
\end{aligned}
$$

Thus we have no associative $\mathrm{SU}(2)$-orbits in the case (1).

Next, consider the case (2). We may assume that $\lambda_{1}=\lambda_{2}$ by Remark 6.4 Then we have $\operatorname{Re}\left(z_{1} \bar{z}_{3}+z_{2} \bar{z}_{4}\right)=0$, and (6.13) implies that

$$
z_{1} \bar{z}_{4}-\bar{z}_{2} z_{3}=0, \quad z_{1} \bar{z}_{3}+\bar{z}_{2} z_{4}=0 \text {. }
$$

Thus,

$$
\begin{aligned}
& z_{1} z_{2} \bar{z}_{3} \bar{z}_{4}=\left|z_{2} z_{3}\right|^{2}=-\left|z_{2} z_{4}\right|^{2}=0 \\
& \bar{z}_{1} \bar{z}_{2} z_{3} z_{4}=\left|z_{1} z_{4}\right|^{2}=-\left|z_{1} z_{3}\right|^{2}=0 .
\end{aligned}
$$

We deduce that either $z_{1}=z_{2}=0$ or $z_{3}=z_{4}=0$. Since ${ }^{t}\left(z_{1}, z_{2}, 0,0\right)$ (resp. $\left.{ }^{t}\left(0,0, z_{3}, z_{4}\right)\right)$ is mapped to ${ }^{t}(1,0,0,0)\left(\operatorname{resp} .^{t}(0,0,1,0)\right)$ by $\bar{z}_{1}-z_{2} j\left(\right.$ resp. $\left.\bar{z}_{3}-z_{4} j\right)$ $\in \operatorname{Sp}(1)_{L}$, we only have to consider at $p_{0}={ }^{t}(1,0,0,0)$ or ${ }^{t}(0,0,1,0)$.

At $p_{0}={ }^{t}(1,0,0,0)$, we have

$$
E_{1}^{*}={ }^{t}(0,0,0,-\sqrt{3}), \quad E_{2}^{*}={ }^{t}(0,0,0, \sqrt{3} i), \quad E_{3}^{*}={ }^{t}(3 i, 0,0,0)=-3 \xi_{1},
$$

which are also orthogonal to each other with respect to $\tilde{g}$ and $\tilde{\varphi}\left(E_{1}^{*}, E_{2}^{*}, E_{3}^{*}\right)=$ $-243 / 25=-\left|E_{1}^{*}\right|_{\tilde{g}}\left|E_{2}^{*}\right|_{\tilde{g}}\left|E_{3}^{*}\right|_{\tilde{g}}$. At $p_{0}={ }^{t}(0,0,1,0)$, we have

$$
E_{1}^{*}={ }^{t}(0,-\sqrt{3}, 0,2), \quad E_{2}^{*}={ }^{t}(0, \sqrt{3} i, 0,2 i), \quad E_{3}^{*}={ }^{t}(0,0,-i, 0)=\xi_{1},
$$

which are also orthogonal to each other with respect to $\tilde{g}$ and $\tilde{\varphi}\left(E_{1}^{*}, E_{2}^{*}, E_{3}^{*}\right)=$ $3^{3} \cdot 19 / 5^{3}=\left|E_{1}^{*}\right|_{\tilde{g}}\left|E_{2}^{*}\right|_{\tilde{g}}\left|E_{3}^{*}\right|_{\tilde{g}}$. Thus we see that both SU(2)-orbits are associative. 


\section{Deformations of homogeneous associative sub- manifolds}

We study the deformations of homogeneous associative submanifolds in the squashed $S^{7}$. We apply the same method of [6] in the standard $S^{7}$.

Proposition 7.1. [6] Let $(Y, \varphi, g)$ be a nearly parallel $G_{2}$-manifold, and $A^{3} \subset Y$ be an associative submanifold. Denote by $\nu$ the normal bundle of $A$ in $Y$ and by $\nabla^{\perp_{A}}$ the connection on $\nu$ induced by the Levi-Civita connection $\nabla$ of $(Y, g)$.

Taking any local orthonormal frame $\left\{e_{1}, e_{2}, e_{3}\right\}$ of $T A$, define the operator $D: C^{\infty}(A, \nu) \rightarrow C^{\infty}(A, \nu)$ by

$$
D \psi:=\sum_{i=1}^{3} e_{i} \times \nabla_{e_{i}}^{\perp_{A}} \psi
$$

Then the vector space of all infinitesimal associative deformations of $A^{3} \hookrightarrow Y$ is identified with $\left\{\psi \in C^{\infty}(A, \nu) ; D \psi=-\psi\right\}$.

Thus to compute the dimensions of the infinitesimal deformation spaces, we only have to know $\nabla^{\perp_{A}}$ and $\times$. The next lemma is useful for the computation.

Lemma 7.2. Let $\left\{e_{1}, e_{2}, e_{3}\right\}$ be the local oriented orthonormal frame of $T A$ satisfying $e_{3}=e_{1} \times e_{2}$. Choose a local normal vector field $V_{1}$ with $\left|V_{1}\right|=1$.

Set $V_{2}=e_{1} \times V_{1}, V_{3}=e_{2} \times V_{1}, V_{4}=-e_{3} \times V_{1}$. Then $\left\{V_{1}, V_{2}, V_{3}, V_{4}\right\}$ is a local orthonormal frame of $\nu$ satisfying

$$
\varphi=e^{123}+e^{1}\left(V^{12}+V^{34}\right)+e^{2}\left(V^{13}+V^{42}\right)-e^{3}\left(V^{14}+V^{23}\right),
$$

where $\left\{e^{i}, V^{j}\right\}$ is a dual coframe of $\left\{e_{i}, V_{j}\right\}$. By the definition of the cross product in Remark 2.7, we have

$$
\left(e_{i} \times V_{j}\right)=\left(\begin{array}{cccc}
V_{2} & -V_{1} & V_{4} & -V_{3} \\
V_{3} & -V_{4} & -V_{1} & V_{2} \\
-V_{4} & -V_{3} & V_{2} & V_{1}
\end{array}\right) .
$$

Lemma 7.3. [6] For any $X, u, v \in \mathfrak{X}(A), \eta \in C^{\infty}(A, \nu)$, we have

$$
\nabla_{X}^{\perp_{A}^{A}}(u \times \eta)=\left(\nabla_{X}^{\top A} u\right) \times \eta+u \times\left(\nabla_{X}^{\perp^{A}} \eta\right)-(\chi(X, u, \eta))^{\perp_{A}},
$$

where $\chi(X, u, \eta)=X \times(u \times \eta)+g(X, u) \eta$ and $\top_{A}: T Y \rightarrow T A$ and $\perp_{A}: T Y \rightarrow \nu$ are projections.

We can compute $\nabla_{e_{i}}^{\perp_{A}} V_{j}$ from $\nabla_{e_{i}}^{\top} e_{j}$ and $\nabla_{e_{i}}^{\perp_{A}} V_{1}$ by Lemma 7.3 and obtain the following. The proof is straightforward and we omit it.

Lemma 7.4. Denote $\nabla_{e_{i}}^{\top} e_{j}=\sum_{k=1}^{3} \Gamma_{i j}^{k} e_{k}$ and $\nabla_{e_{i}}^{\perp^{A}} V_{1}=\sum_{j=2}^{4} K_{i j} V_{j}$. Then we have for $i=1,2,3$

$$
\begin{aligned}
& \nabla_{e_{i}}^{\perp_{A}^{A}} V_{2}=-K_{i 2} V_{1}+\left(\Gamma_{i 1}^{2}-K_{i 4}+\delta_{i 3}\right) V_{3}+\left(-\Gamma_{i 1}^{3}+K_{i 3}+\delta_{i 2}\right) V_{4}, \\
& \nabla_{e_{i}}^{\perp_{A}} V_{3}=-K_{i 3} V_{1}+\left(\Gamma_{i 2}^{1}+K_{i 4}-\delta_{i 3}\right) V_{2}+\left(-\Gamma_{i 2}^{3}-K_{i 2}-\delta_{i 1}\right) V_{4}, \\
& \nabla_{e_{i}}^{\perp_{A}} V_{4}=-K_{i 4} V_{1}+\left(-\Gamma_{i 3}^{1}-K_{i 3}-\delta_{i 2}\right) V_{3}+\left(-\Gamma_{i 3}^{2}+K_{i 2}+\delta_{i 1}\right) V_{3} .
\end{aligned}
$$


By the definition of the Levi-Civita connection, we have the following. The proof is also straightforward and we omit it.

Lemma 7.5. Suppose that $A$ is a Lie group $G$ and $\left\{e_{i}\right\}_{i=1,2,3}$ are left invariant vector fields. Denoting $\left[e_{i}, e_{j}\right]=\sum_{i=1}^{3} c_{i j}^{k} e_{k}\left(c_{i j}^{k} \in \mathbb{R}\right)$, we have

$$
\nabla_{e_{i}}^{\top_{A}} e_{j}=\frac{1}{2} \sum_{k=1}^{3}\left(c_{i j}^{k}-c_{i k}^{j}-c_{j k}^{i}\right) e_{k} .
$$

\subsection{Computations on $\mathrm{SU}(2)$}

For the convenience of the computation, we summarize formulas on $\mathrm{SU}(2)$. Define the basis $\left\{E_{1}, E_{2}, E_{3}\right\}$ of $\mathfrak{s u}(2)$ as 6.51 .

Lemma 7.6. Let $V_{n}$ be a $\mathbb{C}$-vector space of all complex homogeneous polynomials with two variables $z_{1}, z_{2}$ of degree $n$, where $n \geq 0$, and define the representation $\rho_{n}: \mathrm{SU}(2) \rightarrow \mathrm{GL}\left(V_{n}\right)$ as

$$
\left(\rho_{n}\left(\begin{array}{cc}
a & -\bar{b} \\
b & \bar{a}
\end{array}\right) f\right)\left(z_{1}, z_{2}\right)=f\left(\left(z_{1}, z_{2}\right)\left(\begin{array}{cc}
a & -\bar{b} \\
b & \bar{a}
\end{array}\right)\right) .
$$

Define the Hermitian inner product $\langle$,$\rangle of V_{n}$ such that

$$
\left\{v_{k}^{(n)}=\frac{1}{\sqrt{k !(n-k) !}} z_{1}^{n-k} z_{2}^{k}\right\}_{0 \leq k \leq n}
$$

is a unitary basis of $V_{n}$. Denoting by $\widehat{\mathrm{SU}(2)}$ the set of all equivalence classes of finite dimensional irreducible representations of $\mathrm{SU}(2)$, we know that $\widehat{\mathrm{SU}(2)}=$ $\left\{\left(V_{n}, \rho_{n}\right) ; n \geq 0\right\}$. Then every $\mathbb{C}$-valued continuous function on $\mathrm{SU}(2)$ is uniformly approximated by the $\mathbb{C}$-linear combination of the following functions:

$$
\left\{\left\langle\rho_{n}(\cdot) v_{i}^{(n)}, v_{j}^{(n)}\right\rangle ; n \geq 0,0 \leq i, j \leq n\right\},
$$

which are mutually orthogonal with respect to the $L_{2}$ inner product.

By a direct computation, we see the following.

Lemma 7.7. Identify $X \in \mathfrak{s u}(2)$ with the left invariant differential operator on $\mathrm{SU}(2)$. For $u=\sum_{l=0}^{n} C_{l} v_{l}^{(n)} \in V_{n}$, set

$$
u^{*}=\sum_{l=0}^{n}(-1)^{n-l} \bar{C}_{n-l} v_{l}^{(n)} \in V_{n} .
$$

Then for any $n \geq 0,0 \leq k, l \leq n, u, v \in V_{n}, X \in \mathfrak{s u}(2)$, we have

$$
\begin{aligned}
X\left\langle\rho_{n}(\cdot) v, u\right\rangle & =\left\langle\rho_{n}(\cdot) d \rho_{n}(X) v, u\right\rangle, \\
\left(d \rho_{n}(X) v\right)\left(z_{1}, z_{2}\right) & =\left(\frac{\partial v}{\partial z_{1}}, \frac{\partial v}{\partial z_{2}}\right){ }^{t} X\left(\begin{array}{c}
z_{1} \\
z_{2}
\end{array}\right), \\
\overline{\left\langle\rho_{n}(\cdot) v_{k}^{(n)}, u\right\rangle} & =(-1)^{k}\left\langle\rho_{n}(\cdot) v_{n-k}^{(n)}, u^{*}\right\rangle,
\end{aligned}
$$




$$
\begin{aligned}
\left(-i E_{1}+E_{2}\right)\left\langle\rho_{n}(\cdot) v_{k}^{(n)}, u\right\rangle & = \begin{cases}2 i \sqrt{(k+1)(n-k)}\left\langle\rho_{n}(\cdot) v_{k+1}^{(n)}, u\right\rangle, & (k<n) \\
0, & (k=n)\end{cases} \\
\left(i E_{1}+E_{2}\right)\left\langle\rho_{n}(\cdot) v_{k}^{(n)}, u\right\rangle & = \begin{cases}2 i \sqrt{k(n-k+1)}\left\langle\rho_{n}(\cdot) v_{k-1}^{(n)}, u\right\rangle, & (k>0) \\
0, & (k=0)\end{cases} \\
i E_{3}\left\langle\rho_{n}(\cdot) v_{k}^{(n)}, u\right\rangle & =(-n+2 k)\left\langle\rho_{n}(\cdot) v_{k}^{(n)}, u\right\rangle .
\end{aligned}
$$

Lemma 7.8. Suppose that $\left\{e_{1}, e_{2}, e_{3}\right\}=\left\{p E_{1}, p E_{2}, q E_{3}\right\}$, where $0 \neq p, q \in \mathbb{R}$, is an oriented orthonormal basis of $\mathfrak{s u}(2)$ for some metric and orientation. Define the differential operator $D_{\lambda, \mu}: C^{\infty}\left(\mathrm{SU}(2), \mathbb{R}^{4}\right) \rightarrow C^{\infty}\left(\mathrm{SU}(2), \mathbb{R}^{4}\right)$ by

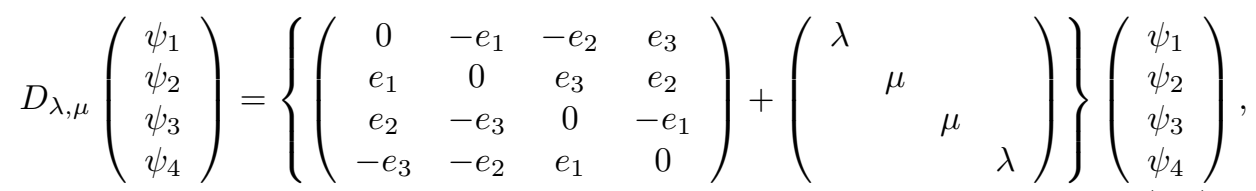

for $\lambda, \mu \in \mathbb{R}$. Setting $\Psi_{1}=\psi_{1}+i \psi_{4}, \Psi_{1}=\psi_{2}-i \psi_{3}, D_{\lambda, \mu}$ is described as

$$
D_{\lambda, \mu}\left(\begin{array}{l}
\Psi_{1} \\
\Psi_{2}
\end{array}\right)=\left\{\left(\begin{array}{cc}
-i e_{3} & -e_{1}-i e_{2} \\
e_{1}-i e_{2} & i e_{3}
\end{array}\right)+\left(\begin{array}{ll}
\lambda & \\
& \mu
\end{array}\right)\right\}\left(\begin{array}{l}
\Psi_{1} \\
\Psi_{2}
\end{array}\right) .
$$

Set $\psi={ }^{t}\left(\psi_{1}, \psi_{2}, \psi_{3}, \psi_{4}\right)$. Then $D_{\lambda, \mu} \psi=\alpha \psi$ for $\alpha \in \mathbb{R}$ is equivalent to

$$
\begin{aligned}
\left(-i e_{3}+\lambda-\alpha\right) \Psi_{1}-\left(e_{1}+i e_{2}\right) \Psi_{2} & =0 \\
\left(e_{1}-i e_{2}\right) \Psi_{1}+\left(i e_{3}+\nu-\alpha\right) \Psi_{2} & =0 .
\end{aligned}
$$

These equations imply that $\Gamma_{p, q, \lambda, \mu, \alpha} \Psi_{2}=0$, where $\Gamma_{p, q, \lambda, \mu, \alpha}$ is defined by

$$
\Gamma_{p, q, \lambda, \mu, \alpha}=\Delta_{+}+\left(\mu-\lambda+2 q-\frac{2 p^{2}}{q}\right) i e_{3}+(-2 q+\lambda-\alpha)(-\mu+\alpha),
$$

where $\Delta_{+}=-\sum_{i=1}^{3} e_{i}^{2}$ is a Laplacian on $\mathrm{SU}(2)$. Especially, for any $n \geq 0,0 \leq$ $k \leq n, u \in V_{n}$, we have

$$
\begin{aligned}
\Delta_{+}\left\langle\rho_{n}(\cdot) v_{k}^{(n)}, u\right\rangle= & \left\{\left(-p^{2}+q^{2}\right)(n-2 k)^{2}+p^{2}\left(n^{2}+2 n\right)\right\}\left\langle\rho_{n}(\cdot) v_{k}^{(n)}, u\right\rangle, \\
\Gamma_{p, q, \lambda, \mu, \alpha}\left\langle\rho_{n}(\cdot) v_{k}^{(n)}, u\right\rangle= & \left\{\left(-p^{2}+q^{2}\right)(n-2 k)^{2}\right. \\
& +p^{2}\left(n^{2}+2 n\right)-\left(q(-\mu+\lambda)+2\left(p^{2}-q^{2}\right)\right)(n-2 k) \\
& +(-2 q+\lambda-\alpha)(-\mu+\alpha)\}\left\langle\rho_{n}(\cdot) v_{k}^{(n)}, u\right\rangle .
\end{aligned}
$$

Remark 7.9. In the case of $\mathrm{SU}(2) / \Gamma$ for some finite subgroup $\Gamma$, we may consider the $\Gamma$ equivariant solutions of (7.2) and (7.3).

Proof. It is straightforward to derive (7.2) and (77.3). Since $\left[e_{1}, e_{2}\right]=\frac{2 p^{2}}{q} e_{3},\left[e_{2}, e_{3}\right]=$ $2 q e_{1},\left[e_{3}, e_{1}\right]=2 q e_{2}$, we have $\left(e_{1}-i e_{2}\right) i e_{3}=\left(i e_{3}+2 q\right)\left(e_{1}-i e_{2}\right)$. Applying $\left(e_{1}-i e_{2}\right)$ to (7.2), we obtain

$$
\left(-i e_{3}-2 q+\lambda-\alpha\right)\left(e_{1}-i e_{2}\right) \Psi_{1}+\left(-e_{1}^{2}-e_{2}^{2}-\frac{2 p^{2}}{q} i e_{3}\right) \Psi_{2}=0 .
$$

Eliminating $\Psi_{1}$ from (7.7) by (7.3) gives (7.4). From Lemma 7.7, we obtain (7.5) and (7.6). 


\subsection{The case $L_{1}$}

Let $\mathrm{SU}(2)=\mathrm{Sp}(1)$ act on $S^{7}$ as (4.1). Then $L_{1}$ is the $\mathrm{SU}(2)$-orbit through $p_{0}={ }^{t}(1,0,0,0)$. Identifying $\mathrm{SU}(2) \ni\left(\begin{array}{cc}a & -\bar{b} \\ b & \bar{a}\end{array}\right) \mapsto a-\bar{b} j \in \mathrm{Sp}(1)$, the vector fields $E_{i}^{*}$ generated by $E_{i} \in \mathfrak{s u}(2)$, where $i=1,2,3$, in (6.5) are described as

$E_{1}^{*}={ }^{t}(1,0,0,0)=-\xi_{2}, \quad E_{2}^{*}={ }^{t}(0, i, 0,0)=-\xi_{3}, \quad E_{3}^{*}={ }^{t}(i, 0,0,0)=-\xi_{1}$,

at $p_{0}$, which induces the orthonormal basis $\left\{e_{1}, e_{2}, e_{3}\right\}=5 / 3\left\{E_{1}, E_{2},-E_{3}\right\}$ of $\mathfrak{s u}(2)$.

Set $v_{1}=\frac{\sqrt{5}}{3} t(0,0,1,0) \in \nu_{p_{0}}$, which is horizontal and $\left|v_{1}\right|_{\tilde{g}}=1$. Denote $X_{0}={ }^{t}(0,0,1,0)$, which is horizontal at $p_{0}$ and $X_{i}=\Phi_{i}\left(X_{0}\right)$ for $i=1,2,3$. By the definition of $\tilde{\varphi}$ in Proposition 4.3 , the vectors $v_{2}=e_{1} \times v_{1}, v_{3}=e_{2} \times v_{1}, v_{4}=$ $-e_{3} \times v_{1}$ are described as $\left\{v_{1}, v_{2}, v_{3}, v_{4}\right\}=\frac{5}{3}\left\{X_{0}, X_{2}, X_{3}, X_{1}\right\}$. Define the vector field $V_{i}$ on $L_{1}$ by $\left(V_{i}\right)_{g \cdot p_{0}}=g_{*} v_{i}$, where $g \in \mathrm{SU}(2)$, we obtain the following by Lemma 4.7 and Lemma 7.5

$$
\left(\begin{array}{c}
\tilde{\nabla}_{e_{1}} V_{1} \\
\tilde{\nabla}_{e_{2}} V_{1} \\
\tilde{\nabla}_{e_{3}} V_{1}
\end{array}\right)=\frac{1}{3}\left(\begin{array}{c}
V_{2} \\
V_{3} \\
-V_{4}
\end{array}\right), \quad\left(\tilde{\nabla}_{e_{i}}^{T^{L_{1}}} e_{j}\right)=\frac{5}{3}\left(\begin{array}{ccc}
0 & -e_{3} & e_{2} \\
e_{3} & 0 & -e_{1} \\
-e_{2} & e_{1} & 0
\end{array}\right) .
$$

This computation and Lemma 7.4 give the following.

$$
\left(\tilde{\nabla}_{e_{i}} V_{j}\right)=\frac{1}{3}\left(\begin{array}{cccc}
V_{2} & -V_{1} & V_{4} & -V_{3} \\
V_{3} & -V_{4} & -V_{1} & V_{2} \\
-V_{4} & -V_{3} & V_{2} & V_{1}
\end{array}\right)
$$

Then by the trivialization of $\nu$ via $\left\{V_{1}, V_{2}, V_{3}, V_{4}\right\}$, we have $D=D_{-1,-1}$, where $D_{\lambda, \mu}$ is defined in (7.1). Using the notations of Lemma 7.8, we see that $\Psi_{2}$ is constant, and hence $\Phi_{1}$ is constant. Thus we obtain $\operatorname{dim}_{\mathbb{R}}\left\{\psi \in C^{\infty}\left(L_{1}, \nu\right) ; D \psi=\right.$ $-\psi\}=4$.

Since $\operatorname{dim}_{\mathbb{R}} \operatorname{Sp}(1) \operatorname{Sp}(2) / \operatorname{Sp}(1)(\operatorname{Sp}(1) \times \operatorname{Sp}(1))=4, \operatorname{Sp}(1) \operatorname{Sp}(2)$ induces 4dimensional associative deformations of $L_{1}$ and we obtain the following.

Proposition 7.10. The associative deformations of $L_{1}$ are trivial. Its deformation space is $\mathrm{Sp}(1) \mathrm{Sp}(2) / \mathrm{Sp}(1)(\mathrm{Sp}(1) \times \mathrm{Sp}(1))=\mathbb{H} P^{1}=S^{4}$. The associative deformations of $L_{1}$ are the deformations of fibers of $\pi: S^{7} \rightarrow S^{4}$ parametrized by the base space $S^{4}$.

\subsection{The case $L_{2}$}

Let $\mathrm{SU}(2)$ act on $S^{7}$ by (6.4). Then $L_{2}$ is the $\mathrm{SU}(2)$-orbit through $p_{0}=$ ${ }^{t}(1,0,0,0)$. By (6.6), the vector fields $E_{i}^{*}$ generated by $E_{i} \in \mathfrak{s u}(2)$ for $i=1,2,3$ in (6.5) are described as

$$
E_{1}^{*}={ }^{t}(0,0,-1,0), \quad E_{2}^{*}={ }^{t}(0,0,-i, 0), \quad E_{3}^{*}={ }^{t}(-i, 0,0,0)=\xi_{1},
$$

and satisfy $\tilde{\varphi}\left(E_{1}^{*}, E_{2}^{*}, E_{3}^{*}\right)=-27 / 25<0$ at $p_{0}$. Then we obtain the induced oriented orthonormal basis $\left\{e_{1}, e_{2}, e_{3}\right\}=\left\{\frac{\sqrt{5}}{3} E_{1}, \frac{\sqrt{5}}{3} E_{2},-\frac{5}{3} E_{3}\right\}$ of $\mathfrak{s u}(2)$.

Set $v_{1}=\frac{5}{3} t(0,1,0,0)=-\frac{5}{3} \xi_{2} \in \nu_{p_{0}}$, which satisfies $\left|v_{1}\right|_{\tilde{g}}=1$. Denote $X_{0}={ }^{t}(0,0,1,0)$, which is horizontal at $p_{0}$ and $X_{i}=\Phi_{i}\left(X_{0}\right)$ for $i=1,2,3$. 
Since $\left\{e_{1}, e_{2}, e_{3}\right\}=\left\{-\frac{\sqrt{5}}{3} X_{0},-\frac{\sqrt{5}}{3} X_{1}, \xi_{1}\right\}$, vectors $v_{2}=e_{1} \times v_{1}, v_{3}=e_{2} \times v_{1}, v_{4}=$ $-e_{3} \times v_{1}$ are described as $\left\{v_{1}, v_{2}, v_{3}, v_{4}\right\}=\left\{-\frac{5}{3} \xi_{2}, \frac{\sqrt{5}}{3} X_{2},-\frac{\sqrt{5}}{3} X_{3},-\frac{5}{3} \xi_{3}\right\}$. Define the vector field $V_{i}$ on $L_{2}$ by $\left(V_{i}\right)_{g \cdot p_{0}}=g_{*} v_{i}$ where $g \in \mathrm{SU}(2)$. As in the case $L_{1}$, we obtain

$$
\left(\tilde{\nabla}_{e_{i}} V_{j}\right)=\frac{1}{3}\left(\begin{array}{cccc}
-V_{2} & V_{1} & -V_{4} & V_{3} \\
-V_{3} & V_{4} & V_{1} & -V_{2} \\
-5 V_{4} & -V_{3} & V_{2} & 5 V_{1}
\end{array}\right) .
$$

Then by the trivialization of $\nu$ via $\left\{V_{1}, V_{2}, V_{3}, V_{4}\right\}$, we have $D=D_{-1,-1 / 3}$, where $D_{\lambda, \mu}$ is defined in (7.1). Setting $(p, q, \lambda, \mu, \alpha)=\left(\frac{\sqrt{5}}{3},-\frac{5}{3},-1, \frac{1}{3},-1\right)$ in (7.6), we see that

$$
\Psi_{2}=\left\langle\rho_{2}(\cdot) v_{1}^{(2)}, u\right\rangle
$$

for $u \in V_{2}$. Since $\operatorname{ker}\left(e_{1}-i e_{2}\right) \cap \operatorname{ker}\left(i e_{3}\right)=\mathbb{C}$, (7.2) and (17.3) imply that

$$
\Psi_{1}=-\frac{\sqrt{10}}{5}\left\langle\rho_{2}(\cdot) v_{2}^{(2)}, u\right\rangle+C
$$

for $C \in \mathbb{C}$. Thus we obtain $\operatorname{dim}_{\mathbb{R}}\left\{\psi \in C^{\infty}\left(L_{2}, \nu\right) ; D \psi=-\psi\right\}=8$.

Since $\operatorname{dim}_{\mathbb{R}} \operatorname{Sp}(1) \operatorname{Sp}(2) / \mathrm{U}(1) \mathrm{U}(2)=8, \operatorname{Sp}(1) \operatorname{Sp}(2)$ induces 8-dimensional associative deformations of $L_{2}$ and we obtain the following.

Proposition 7.11. The associative deformations of $L_{2}$ are trivial. Its deformation space is $\mathrm{Sp}(1) \mathrm{Sp}(2) / \mathrm{U}(1) \mathrm{U}(2)$.

\subsection{The case $A_{1}$}

Let $T^{3}$ act on $S^{7}$ by (6.1). Then $A_{1}$ is the $T^{3}$-orbit through $p_{0}=\frac{1}{2}{ }^{t}(1,1,1, i)$. By (6.3), the vector fields $F_{i}^{*}$ generated by $F_{i}$ for $i=1,2,3$ in (6.2) are described as

$$
F_{1}^{*}=\frac{1}{2} t(i, i, i,-1)=-\xi_{1}, \quad F_{2}^{*}=\frac{1}{2} t^{t}(i,-i, 0,0), \quad F_{3}^{*}=\frac{1}{2} t^{t}(0,0, i, 1),
$$

and satisfy $\tilde{\varphi}\left(F_{1}^{*}, F_{2}^{*}, F_{3}^{*}\right)=-81 / 250<0$ at $p_{0}$. Then we obtain the induced oriented orthonormal basis $\left\{e_{1}, e_{2}, e_{3}\right\}=\left\{\frac{5}{3} F_{1}, \frac{5 \sqrt{6}}{9} F_{2},-\frac{5 \sqrt{6}}{9} F_{3}\right\}$ of $\mathfrak{t}^{3}$.

Set $v_{1}=\frac{\sqrt{5} t}{6}(-1,-1,1, i)$, which is horizontal at $p_{0}$ and $\left|v_{1}\right|_{\tilde{g}}=1$. Denote $X_{0}=\frac{1}{2}{ }^{t}(-1,-1,1, i)$, which is horizontal at $p_{0}$ and $X_{i}=\Phi_{i}\left(X_{0}\right)$ for $i=1,2,3$. Since

$$
e_{1}=-\frac{5}{3} \xi_{1}, \quad e_{2}=\frac{5 \sqrt{6}}{18}\left(\xi_{3}+X_{3}\right), \quad e_{3}=\frac{5 \sqrt{6}}{18}\left(\xi_{2}-X_{2}\right),
$$

vectors $v_{2}=e_{1} \times v_{1}, v_{3}=e_{2} \times v_{1}, v_{4}=-e_{3} \times v_{1}$ are described as

$$
\left\{v_{1}, v_{2}, v_{3}, v_{4}\right\}=\left\{\frac{\sqrt{5}}{3} X_{0}, \frac{\sqrt{5}}{3} X_{1}, \frac{\sqrt{30}}{18}\left(-X_{3}+5 \xi_{3}\right), \frac{\sqrt{30}}{18}\left(X_{2}+5 \xi_{2}\right)\right\} .
$$

Define the vector field $V_{i}$ on $T^{3}$ by $\left(V_{i}\right)_{g \cdot p_{0}}=g_{*} v_{i}$, where $g \in T^{3}$. As in the case $L_{1}$, we obtain

$$
\left(\tilde{\nabla}_{e_{i}}^{\perp_{A_{1}}} V_{j}\right)=\frac{1}{9}\left(\begin{array}{cccc}
3 V_{2} & -3 V_{1} & -12 V_{4} & 12 V_{3} \\
-2 V_{3} & 7 V_{4} & 2 V_{1} & -7 V_{2} \\
2 V_{4} & 7 V_{3} & -7 V_{2} & -2 V_{1}
\end{array}\right) .
$$


Then by the trivialization of $\nu$ via $\left\{V_{1}, V_{2}, V_{3}, V_{4}\right\}$, we have

$$
D=\left(\begin{array}{cccc}
0 & -e_{1} & -e_{2} & e_{3} \\
e_{1} & 0 & e_{3} & e_{2} \\
e_{2} & -e_{3} & 0 & -e_{1} \\
-e_{3} & -e_{2} & e_{1} & 0
\end{array}\right)+\frac{1}{9}\left(\begin{array}{cccc}
1 & & & \\
& 11 & & \\
& & 21 & \\
& & & 21
\end{array}\right) \text {. }
$$

Suppose $D \psi=-\psi$, where $\psi={ }^{t}\left(\psi_{1}, \psi_{2}, \psi_{3}, \psi_{4}\right)$ and $\psi_{i} \in C^{\infty}\left(T^{3}\right)$. Eliminating $\psi_{2}$ by $\psi_{2}=-\frac{9}{20}\left(e_{1}\left(\psi_{1}\right)+e_{3}\left(\psi_{3}\right)+e_{2}\left(\psi_{4}\right)\right)$, we obtain

$$
\begin{aligned}
& \left(\frac{10}{9}+\frac{9}{20} e_{1}^{2}\right) \psi_{1}+\left(\frac{9}{20} e_{1} e_{3}-e_{2}\right) \psi_{3}+\left(\frac{9}{20} e_{1} e_{2}+e_{3}\right) \psi_{4}=0, \\
& \left(\frac{9}{20} e_{1} e_{3}+e_{2}\right) \psi_{1}+\left(\frac{10}{3}+\frac{9}{20} e_{3}^{2}\right) \psi_{3}+\left(\frac{9}{20} e_{2} e_{3}-e_{1}\right) \psi_{4}=0, \\
& \left(\frac{9}{20} e_{1} e_{2}-e_{3}\right) \psi_{1}+\left(\frac{9}{20} e_{2} e_{3}+e_{1}\right) \psi_{3}+\left(\frac{10}{3}+\frac{9}{20} e_{2}^{2}\right) \psi_{4}=0 .
\end{aligned}
$$

Define the smooth function $f_{\gamma} \in C^{\infty}\left(T^{3}, \mathbb{C}\right)$ for $\gamma=\left(\gamma_{1}, \gamma_{2}, \gamma_{3}\right) \in \mathbb{Z}^{3}$ on $T^{3} \cong(\mathbb{R} / 2 \pi \mathbb{Z})^{3}$ by $f_{\gamma}\left(\theta_{1}, \theta_{2}, \theta_{3}\right)=\exp \left(i \sum_{j=1}^{3} \gamma_{j} \theta_{j}\right)$. Identifying $e_{i} \in \mathfrak{t}^{3}$ with the left invariant differential operator on $T^{3}$, we have

$$
e_{1}\left(f_{\gamma}\right)=\frac{5}{3} \gamma_{1} i f_{\gamma}, \quad e_{2}\left(f_{\gamma}\right)=\frac{5 \sqrt{6}}{9} \gamma_{2} i f_{\gamma}, \quad e_{3}\left(f_{\gamma}\right)=-\frac{5 \sqrt{6}}{9} \gamma_{3} i f_{\gamma} .
$$

By a Fourier series expansion, set

$$
\psi_{1}=\sum_{\gamma \in \mathbb{Z}^{3}} C_{\gamma} f_{\gamma}, \quad \psi_{2}=\sum_{\gamma \in \mathbb{Z}^{3}} D_{\gamma} f_{\gamma}, \quad \psi_{3}=\sum_{\gamma \in \mathbb{Z}^{3}} E_{\gamma} f_{\gamma},
$$

where $C_{\gamma}, D_{\gamma}, E_{\gamma} \in \mathbb{C}$. Then (7.8), (17.9), and (17.10) are equivalent to $M_{\gamma}{ }^{t}\left(C_{\gamma}, D_{\gamma}, E_{\gamma}\right)=$ 0 , where

$$
M_{\gamma}=\left(\begin{array}{ccc}
8-9 \gamma_{1}^{2} & 3 \sqrt{6} \gamma_{1} \gamma_{3}-4 \sqrt{6} \gamma_{2} i & -3 \sqrt{6} \gamma_{1} \gamma_{2}-4 \sqrt{6} \gamma_{3} i \\
3 \sqrt{6} \gamma_{1} \gamma_{3}+4 \sqrt{6} \gamma_{2} i & -6 \gamma_{3}^{2}+24 & 6 \gamma_{2} \gamma_{3}-12 \gamma_{1} i \\
-3 \sqrt{6} \gamma_{1} \gamma_{2}+4 \sqrt{6} \gamma_{3} i & 6 \gamma_{2} \gamma_{3}+12 \gamma_{1} i & -6 \gamma_{2}^{2}+24
\end{array}\right)
$$

To obtain a nontrivial solution ${ }^{t}\left(C_{\gamma}, D_{\gamma}, E_{\gamma}\right) \neq 0$,

$$
\operatorname{det} M_{\gamma}=16\left\{\left(9 \gamma_{1}^{2}+6 \gamma_{2}^{2}+6 \gamma_{3}^{2}-22\right)^{2}+4\left(12\left(\gamma_{2}^{2}+\gamma_{3}^{2}\right)-49\right)\right\}
$$

must vanish. We see that $\operatorname{det} M_{\gamma}=0$ if and only if

$$
\left(\gamma_{1}, \gamma_{2}, \gamma_{3}\right)= \pm(2,0,0), \pm(0,2,0), \pm(0,0,2), \pm(0,1,1), \pm(0,1,-1) .
$$

For each $\gamma$ in (17.11), we can check $\operatorname{dim} \operatorname{ker} M_{\gamma}=1$. Moreover, we have $C_{\gamma}=$ $\overline{C_{-\gamma}}, D_{\gamma}=\overline{D_{-\gamma}}$, and $E_{\gamma}=\overline{E_{-\gamma}}$ so that every $\psi_{j}$ is $\mathbb{R}$-valued. Hence we obtain $\operatorname{dim}_{\mathbb{R}}\left\{\psi \in C^{\infty}\left(A_{1}, \nu\right) ; D \psi=-\psi\right\}=10$.

Since $\operatorname{dim}_{\mathbb{R}} \operatorname{Sp}(1) \operatorname{Sp}(2) / T^{3}=10, \operatorname{Sp}(1) \operatorname{Sp}(2)$ induces 10-dimensional associative deformations of $A_{1}$ and we obtain the following.

Proposition 7.12. The associative deformations of $A_{1}$ are trivial. Its deformation space is $\operatorname{Sp}(1) \operatorname{Sp}(2) / T^{3}$. 


\subsection{The case $A_{2}$}

Let $\mathrm{SU}(2)$ act on $S^{7}$ by (6.9). Then $A_{2}$ is the $\mathrm{SU}(2)$-orbit through $p_{0}=$ ${ }^{t}(1,0,0,0)$. By (6.15), $\left\{e_{1}, e_{2}, e_{3}\right\}=\left\{\frac{\sqrt{15}}{9} E_{1}, \frac{\sqrt{15}}{9} E_{2},-\frac{5}{9} E_{3}\right\}$ is the induced oriented orthonormal basis of $\mathfrak{s u}(2)$, where $E_{i} \in \mathfrak{s u}(2)$ for $i=1,2,3$ is defined in (6.5).

Set $v_{1}=\frac{5}{3}^{t}(0,1,0,0)=-\frac{5}{3} \xi_{2} \in \nu_{p_{0}}$, which satisfies $\left|v_{1}\right|_{\tilde{g}}=1$. Denote $X_{0}={ }^{t}(0,0,0,1)$, which is horizontal at $p_{0}$ and $X_{i}=\Phi_{i}\left(X_{0}\right)$ for $i=1,2,3$. Since

$$
e_{1}=-\frac{\sqrt{5}}{3} X_{0}, \quad e_{2}=\frac{\sqrt{5}}{3} X_{1}, \quad e_{3}=-\frac{5}{3} \xi_{1},
$$

vectors $v_{2}=e_{1} \times v_{1}, v_{3}=e_{2} \times v_{1}, v_{4}=-e_{3} \times v_{1}$ are described as

$$
\left\{v_{1}, v_{2}, v_{3}, v_{4}\right\}=\left\{-\frac{5}{3} \xi_{2}, \frac{\sqrt{5}}{3} X_{2}, \frac{\sqrt{5}}{3} X_{3}, \frac{5}{3} \xi_{3}\right\} .
$$

Define the vector field $V_{i}$ in the neighborhood of $p_{0}$ of $A_{2}$ by $\left(V_{i}\right)_{g \cdot p_{0}}=g_{*} v_{i}$, where $g \in \mathrm{SU}(2)$. As in the case $L_{1}$, we obtain

$$
\left(\tilde{\nabla}_{e_{i}}^{\perp_{A_{2}}} V_{j}\right)=\frac{1}{9}\left(\begin{array}{cccc}
-3 V_{2} & 3 V_{1} & -3 V_{4} & 3 V_{3} \\
-3 V_{3} & 3 V_{4} & 3 V_{1} & -3 V_{2} \\
-15 V_{4} & 17 V_{3} & -17 V_{2} & 15 V_{1}
\end{array}\right) .
$$

Then by the local trivialization of $\nu$ via $\left\{V_{1}, V_{2}, V_{3}, V_{4}\right\}$, we have $D=D_{-1,23 / 9}$, where $D_{\lambda, \mu}$ is defined in (7.1). Setting $(p, q, \lambda, \mu, \alpha)=\left(\frac{\sqrt{15}}{9},-\frac{5}{9},-1, \frac{23}{9},-1\right)$ in (17.6), we see that

$$
\Psi_{2}=\left\langle\rho_{6}(\cdot) v_{5}^{(6)}, u\right\rangle
$$

for $u \in V_{2}$. Since $\operatorname{ker}\left(e_{1}-i e_{2}\right) \cap \operatorname{ker}\left(i e_{3}\right)=\mathbb{C}$, (7.2) and (17.3) imply that

$$
\Psi_{1}=-\frac{\sqrt{10}}{5}\left\langle\rho_{6}(\cdot) v_{6}^{(6)}, u\right\rangle+C
$$

for $C \in \mathbb{C}$. These solutions are $\mathbb{Z}_{3}$-equivariant, and hence we obtain $\operatorname{dim}_{\mathbb{R}}\{\psi \in$ $\left.C^{\infty}\left(A_{2}, \nu\right) ; D \psi=-\psi\right\}=16$

Since $\operatorname{dim}_{\mathbb{R}} \operatorname{Sp}(1) \operatorname{Sp}(2) / \mathrm{U}(1) \mathrm{SU}(2)=9, \operatorname{Sp}(1) \operatorname{Sp}(2)$ induces 9-dimensional associative deformations of $A_{2}$. Thus $A_{2}$ can have at most 7-dimensional family of nontrivial associative deformations. In fact, we obtain the following.

Proposition 7.13. All associative deformations of $A_{2}$ are induced by the $\operatorname{Sp}(1) \operatorname{Sp}(2)$ action and by the $\operatorname{PSp}(2, \mathbb{C})$-action on $\mathbb{C} P^{3}$ via the Hopf lift. In other words, all the associative deformations of $A_{2}$ are given by the following.

- the $\operatorname{PSp}(2, \mathbb{C})$-action on $\mathbb{C} P^{3}$ via the Hopf lift, which corresponds to the deformation of $p_{1}\left(A_{2}\right)$ as a horizontal holomorphic curve, where $p_{1}: S^{7} \rightarrow$ $\mathbb{C} P^{3}$ is a projection,

- the action generated by $j, k \in \mathrm{Sp}(1)$.

Note that $\operatorname{PSp}(2, \mathbb{C})$ acts on $\mathbb{C} P^{3}$ as the group of biholomorphic maps which preserve the horizontal distribution [3, [10. 
Proof. First description is an analogue of [10, 6] and we omit the proof. The second description follows from the next lemma.

Lemma 7.14. The subgroup of $\operatorname{PSp}(2, \mathbb{C})$ which preserves $p_{1}\left(A_{2}\right)$ is isomorphic to $\operatorname{PSL}(2, \mathbb{C})$. Thus the deformation space of $p_{1}\left(A_{2}\right)$ as a holomorphic curve is $\operatorname{PSp}(2, \mathbb{C}) / \operatorname{PSL}(2, \mathbb{C})$, which is 14-dimensional.

Proof. The inclusion $\mathrm{SU}(2) \hookrightarrow \mathrm{Sp}(2)$ of $(6.9)$ is canonically extended to $G L(2, \mathbb{C}) \hookrightarrow$ $\operatorname{GL}(4, \mathbb{C})$ :

$\left(g_{i j}\right) \mapsto\left(\begin{array}{cccc}g_{11}^{3} & g_{12}^{3} & \sqrt{3} g_{11} g_{12}^{2} & \sqrt{3} g_{11}^{2} g_{12} \\ g_{21}^{3} & g_{22}^{3} & \sqrt{3} g_{21} g_{22}^{2} & \sqrt{3} g_{21}^{2} g_{22} \\ \sqrt{3} g_{11} g_{21}^{2} & \sqrt{3} g_{12} g_{22}^{2} & g_{22}\left(g_{11} g_{22}+2 g_{12} g_{21}\right) & g_{21}\left(2 g_{11} g_{22}+g_{12} g_{21}\right) \\ \sqrt{3} g_{11}^{2} g_{21} & \sqrt{3} g_{12}^{2} g_{22} & g_{12}\left(2 g_{11} g_{22}+g_{12} g_{21}\right) & g_{11}\left(g_{11} g_{22}+2 g_{12} g_{21}\right)\end{array}\right)$,

which is the group of biholomorphic maps which preserve $p_{1}\left(A_{2}\right)$. We can check that $G L(2, \mathbb{C}) \cap \operatorname{Sp}(2, \mathbb{C})=\operatorname{SL}(2, \mathbb{C})$, and hence we obtain the proof.

\subsection{The case $A_{3}$}

Let $\mathrm{SU}(2)$ act on $S^{7}$ by (6.9). Then $A_{3}$ is the $\mathrm{SU}(2)$-orbit through $p_{0}=$ ${ }^{t}(0,0,1,0)$. By (6.16), $\left\{e_{1}, e_{2}, e_{3}\right\}=\left\{\frac{5 \sqrt{19}}{57} E_{1}, \frac{5 \sqrt{19}}{57} E_{2}, \frac{5}{3} E_{3}\right\}$ is the induced oriented orthonormal basis of $\mathfrak{s u}(2)$, where $E_{i} \in \mathfrak{s u}(2)$ for $i=1,2,3$ is defined in (6.5).

Set $v_{1}=\frac{\sqrt{5}}{3}^{t}(1,0,0,0) \in \nu_{p_{0}}$, which is horizontal at $p_{0}$ and $\left|v_{1}\right|_{\tilde{g}}=1$. Denote $X_{0}={ }^{t}(1,0,0,0)$, which is horizontal at $p_{0}$ and $X_{i}=\Phi_{i}\left(X_{0}\right)$ for $i=1,2,3$. Since

$$
e_{1}=-\frac{5 \sqrt{19}}{57}\left(2 \xi_{2}+\sqrt{3} X_{2}\right), \quad e_{2}=\frac{5 \sqrt{19}}{57}\left(-2 \xi_{3}+\sqrt{3} X_{3}\right), \quad e_{3}=\frac{5}{3} \xi_{1},
$$

vectors $v_{2}=e_{1} \times v_{1}, v_{3}=e_{2} \times v_{1}, v_{4}=-e_{3} \times v_{1}$ are described as

$$
\left\{v_{1}, v_{2}, v_{3}, v_{4}\right\}=\left\{\frac{\sqrt{5}}{3} X_{0}, \frac{\sqrt{95}}{57}\left(-5 \sqrt{3} \xi_{2}+2 X_{0}\right), \frac{\sqrt{95}}{57}\left(5 \sqrt{3} \xi_{3}+2 X_{3}\right), \frac{\sqrt{5}}{3} X_{1}\right\} \text {. }
$$

Define the vector field $V_{i}$ on $\mathrm{SU}(2)$ by $\left(V_{i}\right)_{g \cdot p_{0}}=g_{*} v_{i}$, where $g \in \mathrm{SU}(2)$. As in the case $L_{1}$, we obtain

$$
\left(\tilde{\nabla}_{e_{i}}^{\perp A_{3}} V_{j}\right)=\frac{1}{57}\left(\begin{array}{cccc}
-31 V_{2} & 31 V_{1} & -31 V_{4} & 31 V_{3} \\
-31 V_{3} & 31 V_{4} & 31 V_{1} & -31 V_{2} \\
361 V_{4} & -119 V_{3} & 119 V_{2} & -361 V_{1}
\end{array}\right)
$$

Then by the local trivialization of $\nu$ via $\left\{V_{1}, V_{2}, V_{3}, V_{4}\right\}$, we have $D=D_{141 / 19,-1}$, where $D_{\lambda, \mu}$ is defined in (7.1). Setting $(p, q, \lambda, \mu, \alpha)=\left(\frac{5 \sqrt{19}}{57}, \frac{5}{3}, \frac{141}{19},-1,-1\right)$ in (17.6), we see that

$$
\Psi_{2}=\left\langle\rho_{6}(\cdot) v_{4}^{(6)}, u\right\rangle+C
$$

for $u \in V_{2}, C \in \mathbb{C}$. Since $\operatorname{ker}\left(i e_{3}-\frac{160}{19}\right)=\{0\}$, (7.2) and (7.3) imply that

$$
\Psi_{1}=-\frac{\sqrt{190}}{10}\left\langle\rho_{6}(\cdot) v_{5}^{(6)}, u\right\rangle .
$$


Hence we obtain $\operatorname{dim}_{\mathbb{R}}\left\{\psi \in C^{\infty}\left(A_{3}, \nu\right) ; D \psi=-\psi\right\}=16$.

Since $\operatorname{dim}_{\mathbb{R}} \operatorname{Sp}(1) \mathrm{Sp}(2) / \mathrm{SU}(2)=10, \mathrm{Sp}(1) \mathrm{Sp}(2)$ induces 10-dimensional associative deformations of $A_{3}$. Thus $A_{3}$ can have at most 6-dimensional family of nontrivial associative deformations.

The associative deformation space of $A_{3}$ is explained by a one-to-one correspondence between null-torsion $I_{1}^{\prime}$-holomorphic curves and horizontal holomorphic curves in $\mathbb{C} P^{3}(12)$.

Decompose $T \mathbb{C} P^{3}=\underline{\mathcal{H}} \oplus \underline{\mathcal{V}}$, where $\underline{\mathcal{V}}$ is a vector bundle tangent to the fibers of $p_{2}: \mathbb{C} P^{3} \rightarrow S^{4}$, and $\underline{\mathcal{H}}$ is its orthogonal complement bundle of $\underline{\mathcal{V}}$. Define a map $P: \underline{\mathcal{H}}-\{0\} \rightarrow \mathbb{C} P^{3}$ by $P(v)=[\tilde{v}]$, where $\tilde{v} \in \mathcal{H} \subset T S^{7}$ is a horizontal lift of $v$ with respect to $p_{1}: S^{7} \rightarrow \mathbb{C} P^{3}$ and we identify $\tilde{v}$ with a vector in $\mathbb{C}^{4}$.

Let $\operatorname{pr}_{\mathcal{H}}: T \mathbb{C} P^{3} \rightarrow \underline{\mathcal{H}}$ be a canonical projection and $\Sigma \subset \mathbb{C} P^{3}$ be a $I_{1}^{\prime}$ holomorphic curve with $\left.p r_{\mathcal{H}}\right|_{T \Sigma} \neq 0$. Then there exist a holomorphic line bundle $\left.L \subset \underline{\mathcal{H}}\right|_{\Sigma}$ such that $\operatorname{pr}_{\mathcal{H}}(\bar{T} \Sigma) \subset L$. If $\operatorname{pr}_{\underline{\mathcal{H}}}$ is nowhere vanishing on $\Sigma, L=$ $\operatorname{pr}_{\underline{\mathcal{H}}}(T \Sigma)$. Denote by $\left.L^{\perp} \underline{\mathcal{H}} \subset \underline{\mathcal{H}}\right|_{\Sigma}$ the orthonormal complement bundle of $L$ and set $\hat{\Sigma}=P\left(L^{\perp \underline{H}}-\{0\}\right)$.

Definition 7.15. A non-vertical $I_{1}^{\prime}$-holomorphic curve $\Sigma$ is called null-torsion if $\hat{\Sigma}$ is a horizontal holomorphic curve.

Proposition 7.16. [12] There is a one-to-one correspondence between nulltorsion $I_{1}^{\prime}$-holomorphic curves and horizontal holomorphic curves via $\Sigma \mapsto \hat{\Sigma}$.

Since $p_{1}\left(A_{3}\right)$ is an image of $\mathbb{C} P^{1}$, it is a null-torsion ([12]). We see the following.

Lemma 7.17. By Proposition 7.16, $p_{1}\left(A_{3}\right)$ corresponds to $p_{1}\left(A_{2}\right)$.

Proof. Since $\operatorname{pr}_{\underline{\mathcal{H}}}$ is nowhere vanishing on $p_{1}\left(A_{3}\right), L=\operatorname{pr}_{\underline{\mathcal{H}}}\left(T\left(p_{1}\left(A_{3}\right)\right)\right)$. By (7.12), $T_{p_{1}\left(p_{0}\right)}\left(p_{1}\left(A_{3}\right)\right)$ is a projection of the subspace of $T_{p_{0}} S^{7}$ spanned by $-2 \xi_{2}-\sqrt{3} X_{2}$ and $-2 \xi_{3}+\sqrt{3} X_{3}$. Thus the vector bundle $\tilde{L}^{\perp}{ }_{\mathcal{H}}$ over $A_{3}$ whose fiber at $g \cdot p_{0}$, where $g \in \mathrm{SU}(2)$, is spanned by $g_{*} X_{0}$ and $g_{*} X_{1}$ satisfies $\left(p_{1}\right)_{*}\left(\tilde{L}^{\perp_{\mathcal{H}}}\right)=$ $L^{\perp_{\underline{H}}}$, which implies that

$$
\begin{aligned}
\widehat{p_{1}\left(A_{3}\right)} & =\left[L^{\perp \underline{H}}-\{0\}\right] \\
& =\left\{\left[g^{t}(1,0,0,0)\right] \in \mathbb{C} P^{3} ; g \in \mathrm{SU}(2)\right\}=p_{1}\left(A_{2}\right) .
\end{aligned}
$$

Remark 7.18. We easily see that $\widehat{p_{1}\left(A_{1}\right)}=p_{1}\left(A_{1}\right)$, and hence $p_{1}\left(A_{1}\right)$ is not null-torsion.

Since the deformation space of $p_{1}\left(A_{2}\right)$ as a horizontal holomorphic curve is 14-dimensional by Proposition 7.13, we obtain the following result.

Proposition 7.19. All the associative deformations of $A_{3}$ are given by the following.

- the Hopf lift of null-torsion $I_{1}^{\prime}$-holomorphic curves, which correspond to horizontal holomorphic curves obtained by deforming $p_{1}\left(A_{2}\right)$ by the $\operatorname{PSp}(2, \mathbb{C})$ action on $\mathbb{C} P^{3}$ by Proposition 7.16 .

- the action generated by $j, k \in \mathrm{Sp}(1)$. 


\section{References}

[1] C. Bär, Real Killing spinors and holonomy, Comm. Math. Phys. 154 (1993), 509-521.

[2] A.L. Besse, Einstein manifolds, Springer-Verlag, New York, 1987.

[3] J. Bolton and L. M. Woodward, Higher singularities and the twistor fibration $\pi: \mathbb{C} P^{3} \rightarrow S^{4}$, Geom. Dedicata 80 (2000), 231-245.

[4] T. Friedrich, I. Kath, A. Moroianu, and U. Semmelmann, On nearly parallel $G_{2}$-structures, J. Geom. Phys. 23 (1997), 259-286.

[5] R. Harvey and H. B. Lawson, Calibrated geometries, Acta Math. 148 (1982), 47-157.

[6] K. Kawai, Deformations of homogeneous associative submanifolds in nearly parallel $G_{2}$-manifolds, math.DG/1407.8046.

[7] J. D. Lotay, Associative Submanifolds of the 7-Sphere, Proc. Lond. Math. Soc. (3) 105 (2012), 1183-1214.

[8] S. P. Marshall, Some Special Lagrangian Submanifolds of $\mathbb{C}^{m}$, dissertation, University of Oxford, 1999.

[9] K. Mashimo, Homogeneous Totally Real Submanifolds of $S^{6}$, Tsukuba J. Math. 9 (1985), 185-202.

[10] Y. Ohnita, On deformation of 3-dimensional certain minimal Legendrian submanifolds, Proceedings of The Thirteenth International Workshop on Diff. Geom. 13 (2009), 71-87.

[11] S. M. Salamon, Riemannian Geometry and Holonomy Groups, Pitman Research Notes in Mathematics 201, Longman, Harlow, 1989.

[12] F. Xu, Pseudoholomorphic curves in nearly Kähler $\mathbf{C P}^{3}$, Differential Geom. Appl. 28, (2010), 107-120.

[13] K. Yano, The theory of Lie derivatives and its applications, North-Holland, Amsterdam, 1957.

Graduate School of Mathematical Sciences, University of Tokyo 3-8-1 Komaba, Meguro, Tokyo 153-8914, Japan

E-mail address: kkawai@ms.u-tokyo.ac.jp 